\title{
A Comparative Study of Molecular Dynamics in Cartesian and in Internal Coordinates: Dynamical Instability in the Latter Caused by Nonlinearity of the Equations of Motion
}

\author{
SANG-HO LEE, KIM PALMO, SAMUEL KRIMM \\ Biophysics Research Division and Department of Physics, University of Michigan, \\ 930 N. University Ave., Ann Arbor, Michigan 48109 \\ Received 18 July 2006; Accepted 13 November 2006 \\ DOI 10.1002/jcc.20627 \\ Published online 5 February 2007 in Wiley InterScience (www.interscience.wiley.com).
}

\begin{abstract}
The stability of a general molecular dynamics (MD) integration scheme is examined for simulations in generalized (internal plus external) coordinates (GCs). An analytic expression is derived for the local error in energy during each integration time step. This shows that the explicit dependence of the mass-matrix on GCs, which makes the system's Lagrange equations of motion nonlinear, causes MD simulations in GCs to be less stable than those in Cartesian coordinates (CCs). In terms of CCs, the corresponding mass-matrix depends only on atomic masses and thus atomistic motion is subject to the linear Newton equations, which makes the system more stable. Also investigated are two MD methods in GCs that utilize nonzero elements of the vibrational spectroscopic B-matrices. One updates positions and velocities in GCs that are iteratively adjusted so as to conform to the velocity Verlet equivalent in GCs. The other updates positions in GCs and velocities in CCs that are adjusted to satisfy the internal constraints of the new constrained WIGGLE MD scheme. The proposed methods are applied to an isolated $n$-octane molecule and their performances are compared with those of several CCMD schemes. The simulation results are found to be consistent with the analytic stability analysis. Finally, a method is presented for computing nonzero elements of B-matrices for external rotations without imposing the Casimir-Eckart conditions.
\end{abstract}

(C) 2007 Wiley Periodicals, Inc. J Comput Chem 28: 1107-1118, 2007

Key words: molecular dynamics; internal coordinate; external rotation; constrained dynamics; numerical integration

\section{Introduction}

Information on the structures and dynamics of biomolecules (e.g., proteins, DNA, and RNA) is basic to an understanding of their biological properties. As a standard coordinate system, Cartesian coordinates (CCs) have been used to visualize detailed atomic level structures and motions. However, molecular internal coordinates (ICs: bond lengths, bond angles, in-plane/out-ofplane wags, or torsion angles) have also been efficiently used in such areas as vibrational (infrared and Raman) normal mode analyses, ${ }^{1-3}$ molecular mechanics (MM) conformation energy analyses, ${ }^{4-6}$ Monte Carlo (MC) simulations, ${ }^{7-15}$ ab initio geometry optimizations, ${ }^{16-30}$ quantum MC simulations, ${ }^{31,32}$ and molecular docking problems. ${ }^{33-37}$ This is because intramolecular interaction energy terms, as well as molecular internal vibrations, are easily described in ICs. However, in contrast to the linearity of the Newton equations of motion in CCs, the classical molecular dynamics (MD) equations to be solved in ICs are not only nonlinear but also demand nontrivial computation processes at each numerical integration step. Thus, MD simulations in ICs have not been as popular as those in CCs.
The equations of motion for typical MD simulations are not analytically solvable, and therefore they are solved approximately by a numerical integration method. To maintain the system's stability, the integration time step routinely has to be kept small enough (less than $1 \mathrm{fs}$ ) to resolve such fast motions as vibrations of bond length ICs. This imposes a serious limitation on routine simulations in a longer time range than ns, where interesting biomolecular conformation changes may take place. However, the fast atomistic motions related to most bond stretching and angle bending vibrations are localized ${ }^{1-3}$ and, during certain simulation time ranges of a molecule, the average changes in the internal bond lengths and bond angles are negligible compared with those in torsion (viz., dihedral) angles. ${ }^{38}$ Thus, freezing (or constraining) all bond length coordinates enables one to use a time step larger than that for an unconstrained CCMD simulation. ${ }^{39,40}$ On the other hand, in the early studies

Correspondence to: S. Krimm; e-mail: skrimm@umich.edu Contract/grant sponsor: NSF; contract/grant number: MCB-0212232 and DMR-0239417 
of conformation energies and dynamics models in ICs, molecules were treated as chains of linked rigid bodies with/without constraints on bond lengths or bond angles. ${ }^{41-45}$ (Interestingly, similar ideas can also be found in mechanical dynamics treatments of complex spacecraft. ${ }^{46,47}$ ) In this approach, since changes in torsion ICs dominate conformational changes, energy minimizations for biomolecules are efficiently carried out first in a space of torsion degrees of freedom and then other ICs are included in a further calculation. This idea has been developed into the torsion angle (or reduced variable) MD method, ${ }^{48-55}$ where all internal degrees of freedom except for torsions are simply neglected. It was further developed to include bond lengths and bond angles, ${ }^{56-60}$ to incorporate quaternion parameters and angular velocities for rotations of linked rigid body subunits, ${ }^{61,62}$ or to adopt the fast recursive algorithm for mechanical models of linked rigid bodies or hinges. ${ }^{63-74}$ In addition to successful applications to structural calculations, ${ }^{67,69,71,74-81}$ reports of using increased integration time steps greater than $4 \mathrm{fs}^{52,68,72,73}$ have made the ICMD method very attractive.

In this development, and based on the spectroscopic B-matrix (viz., the transformation matrix from quantities in $\mathrm{CCs}$ to those in ICs), ${ }^{1,2}$ we have also introduced an ICMD scheme. ${ }^{82,83}$ The efficiency of this scheme lies in the fact that inversion of the large mass-matrix in solving the nonlinear equations of motion is indirectly accomplished by computing the sparse B-matrix elements, which are nonzero only for a few (at most four) related atoms for each IC. In this article, we report a further refinement of the B-matrix ICMD method based on WIGGLE (a new constrained CCMD scheme) ${ }^{84}$ the velocity update in CCs by Pulay and Paizs, ${ }^{85}$ and an improved computation of B-matrix elements for external rotations [see Appendix]. The method is applied to an isolated octane molecule, and its performance (with/without constraints on internal bond lengths) is compared with that of the CCMD method. Mazur made detailed comparative studies between ICMD and CCMD simulations, ${ }^{60,86}$ and found no essential differences between the two MD trajectories. However, we have found that the ICMD method is inherently an order of the integration time step less stable than the CCMD method with/without internal constraints. This is consistent with our detailed theoretical analysis of the two dynamic stabilities. Our derived analytic expression for energy drift in each integration time step is also consistent with that of Gibson and Scheraga. ${ }^{52}$ Overall, this result indicates that, for the purpose of MD simulation itself, CCs are to be preferred to ICs with/without internal constraints. ${ }^{87}$

In Dynamical Equations of Motion in ICs with Internal Constraints, equations of motion in generalized (internal plus external) coordinates (GCs) with internal constraints are introduced. In Local Energy Drift, to analyze the stability of a general ICMD scheme, we investigate the extent to which the system conserves its total energy in an integration time step. In ICMD Algorithms, we introduce two optimal constrained ICMD schemes: AICMD adopts an iterative treatment to conform to the velocity Verlet ${ }^{88}$ equivalent in GCs, and BICMD incorporates the velocity update in $\mathrm{CCs}^{85}$ based on WIGGLE. ${ }^{84}$ In Application to an $n$-Octane Molecule, the two schemes are applied to an isolated octane molecule and their results are compared with those from several CCMD methods with/without con- straints on all $\mathrm{CH}$ bond lengths. Finally, an improved method of computing nonzero B-matrix elements for external rotations is presented in the Appendix.

\section{Dynamical Equations of Motion in ICs with Internal Constraints}

Without a loss of generality, we consider an isolated molecule of $p$ atoms whose masses and Cartesian positions are given by $m_{\lambda}$ and $\mathbf{x}_{\lambda}(\lambda=1, \ldots, p)$, respectively. We define $\mathbf{X} \equiv\left(x_{1}{ }^{1}\right.$ $\left.x_{1}^{2} x_{1}^{3} \ldots x_{\mathrm{p}}{ }^{1} x_{\mathrm{p}}{ }^{2} x_{\mathrm{p}}{ }^{3}\right)^{T}$, with superscript $T$ representing the transpose of a matrix. Let $\mathbf{S}$ be a nonredundant set of GCs such that, in the neighborhood of an arbitrary molecular configuration $\mathbf{S}_{\mathrm{ec}}$ (expansion center),

$$
\begin{aligned}
& \mathbf{S}=\mathbf{S}_{\mathrm{ec}}+\mathbf{B} \Delta \mathbf{X}+\frac{1}{2} \Delta \mathbf{X}^{T} \mathbf{B}_{2} \Delta \mathbf{X}+\cdots \\
& \mathbf{X}=\mathbf{X}_{\mathrm{ec}}+\mathbf{A} \Delta \mathbf{S}+\frac{1}{2} \Delta \mathbf{S}^{T} \mathbf{A}_{2} \Delta \mathbf{S}+\cdots
\end{aligned}
$$

with $\Delta \mathbf{S} \equiv \mathbf{S}-\mathbf{S}_{\mathrm{ec}}, \Delta \mathbf{X} \equiv \mathbf{X}-\mathbf{X}_{\mathrm{ec}}, \mathbf{B} \equiv[\partial \mathbf{S} / \partial \mathbf{X}]_{\mathrm{ec}} \equiv\left[\partial_{\mathbf{X}} \mathbf{S}\right]_{\mathrm{ec}}$, $\mathbf{B}_{2} \equiv\left[\partial_{\mathbf{X}} \partial_{\mathbf{X}} \mathbf{S}\right]_{\mathrm{ec}} \equiv\left[\partial_{\mathbf{X}}^{2} \mathbf{S}\right]_{\mathrm{ec}}, \mathbf{A} \equiv\left[\partial_{\mathbf{S}} \mathbf{X}\right]_{\mathrm{ec}}, \mathbf{A}_{2} \equiv\left[\partial_{\mathbf{S}}^{2} \mathbf{X}\right]_{\mathrm{ec}}$, and

$$
\mathbf{A B}=\mathbf{1}=\mathbf{B} \mathbf{A}\left(\sum_{\beta} A_{\beta}^{\nu^{j}} B_{\lambda^{k}}^{\beta}=\delta_{\lambda}^{\nu} \delta_{k}^{j} \text { and } \delta_{\gamma}^{\beta}=\sum_{\nu=1}^{p} \sum_{j=1}^{3} B_{\nu^{j}}^{\beta} A_{\gamma}^{\nu^{j}}\right) .
$$

Differentiating eq. (3), we have

$$
\mathbf{B}^{T} \mathbf{A}_{2} \mathbf{B}+\mathbf{A} \mathbf{B}_{2}=\mathbf{0}=\mathbf{A}^{T} \mathbf{B}_{2} \mathbf{A}+\mathbf{B} \mathbf{A}_{2} .
$$

In using this simplified expression, we have to keep in mind that $\mathbf{B}_{2}$ and $\mathbf{A}_{2}$ are not regular matrices but third rank tensors. The expressions with specific tensor components corresponding to eq. (4) can be obtained by considering the expressions in the parenthesis of eq. (3). Thus, eqs. (1) and (2) are inverse relations to each other if eqs. (3) and (4) are satisfied. Specific methods of computing elements of $\mathbf{B}, \mathbf{B}_{2}, \mathbf{A}$, and $\mathbf{A}_{2}$ can be found elsewhere. ${ }^{1,2,48,53,56,82,83,89}$ We have verified the correctness of analytical formulas for nonzero elements of $\mathbf{B}$ and $\mathbf{B}_{2}$ by comparing results from numerical and analytical differentiations.

The system's classical kinetic energy $T$ is now expressed by

$$
2 T=\dot{\mathbf{X}}^{T} \mathbf{m} \dot{\mathbf{X}}=\dot{\mathbf{S}}^{T} \mathbf{g S}
$$

where a dot represents differentiation with respect to time and $\mathbf{g}$ $\equiv \mathbf{A}^{T} \mathbf{m A}$ is the mass-matrix, with $\mathbf{m}$ being a $3 p \times 3 p$ diagonal matrix containing triads of atomic masses $m_{\lambda}$. In the presence of $N_{\mathrm{c}}$ nonredundant internal constraints $\sigma^{\alpha}(\mathbf{X})=\mathrm{C}^{\alpha}$ (constant) $\left(\alpha=1, \ldots, N_{\mathrm{c}}\right)$, which are represented by

$$
\boldsymbol{\sigma}(\mathbf{X})=\mathbf{C},
$$


classical atomic motion is subject to the constrained potential energy

$$
V_{\mathrm{c}} \equiv V+\boldsymbol{\sigma}^{T} \Lambda
$$

where $V$ is the system's potential energy without constraints and $\Lambda$ is a column vector of the Lagrange undetermined parameters related to the constraint forces. Assuming $V_{\mathrm{c}}$ is not an explicit function of time and atomic velocities, the classical equations of motion in CCs are found to be

$$
\mathbf{m} \ddot{\mathbf{X}}=-\partial_{\mathbf{X}} V_{\mathrm{c}}=\mathbf{F}_{X}-\mathbf{B}_{\mathrm{c}}^{T} \Lambda
$$

with $\mathbf{F}_{X} \equiv-\partial_{\mathbf{X}} \mathrm{V}$ and $\mathbf{B}_{\mathrm{c}} \equiv \partial \boldsymbol{\sigma} / \partial \mathbf{X} \equiv \partial_{\mathbf{X}} \boldsymbol{\sigma}$. Dynamic equations of motion without constraints are simply obtained by setting $\Lambda$ $=\mathbf{0}$. Successive differentiations of eq. (6) with respect to time provide additional equations for constraints:

$$
\begin{gathered}
\dot{\boldsymbol{\sigma}}=\mathbf{B}_{\mathrm{c}} \dot{\mathbf{X}}=\mathbf{0} \\
\ddot{\boldsymbol{\sigma}}=\mathbf{B}_{\mathrm{c}} \ddot{\mathbf{X}}+\dot{\mathbf{X}}^{T} \mathbf{B}_{2 \mathrm{c}} \dot{\mathbf{X}}=\mathbf{0}
\end{gathered}
$$

with $\mathbf{B}_{2 \mathrm{c}} \equiv \partial_{\mathbf{X}} \mathbf{B}_{\mathrm{c}} \equiv \partial_{\mathbf{X}}^{2} \boldsymbol{\sigma}$. By using eqs. (8) and (10), for given $\dot{\mathbf{X}}$ and $\mathbf{F}_{X}$, the parameters $\Lambda$ can be determined by solving

$$
\mathbf{G}_{\mathrm{cc}} \Lambda=\mathbf{B}_{\mathrm{c}} \mathbf{m}^{-1} \mathbf{F}_{X}+\dot{\mathbf{X}}^{T} \mathbf{B}_{2 \mathrm{c}} \dot{\mathbf{X}}
$$

where $\mathbf{G}_{\mathrm{cc}} \equiv \mathbf{B}_{\mathrm{c}} \mathbf{m}^{-1} \mathbf{B}_{\mathrm{c}}{ }^{T}$, with superscript -1 representing the inverse of a nonsingular matrix. In terms of the GCs, the EulerLagrange equations of motion are found to be

$$
\mathbf{g} \ddot{\mathbf{S}}+\dot{\mathbf{S}}^{T}\left[\mathbf{A}^{T} \mathbf{m} \mathbf{A}_{2}\right] \dot{\mathbf{S}}+\partial_{\mathbf{S}} V_{\mathrm{c}}=\mathbf{0}
$$

with the simplified second term being defined by

$$
\dot{\mathbf{S}}^{T}\left[\mathbf{A}^{T} \mathbf{m} \mathbf{A}_{2}\right] \dot{\mathbf{S}} \equiv\left[\mathbf{A}^{T} \mathbf{m}\left(\dot{\mathbf{S}}^{T} \partial_{\mathbf{S}}\right) \mathbf{A}\right] \dot{\mathbf{S}}=\frac{1}{2}\left[\left(\dot{\mathbf{S}}^{T} \partial_{\mathbf{S}}\right) \mathbf{g}\right] \dot{\mathbf{S}}
$$

Dynamics trajectory information can be obtained from solving eq. (12). For systems of polyatomic molecules, this is done by numerical integration with a finite time step.

As a way of increasing the time step in MD simulations, it has been common to constrain such fast moving degrees of freedom as bond length ICs. ${ }^{39,40}$ We consider constraining some coordinates of $\mathbf{S}$ so that $\mathbf{S}$ can be separated into an unconstrained part $\mathbf{S}_{\mathrm{u}}$ and a constrained one $\mathbf{S}_{\mathrm{c}}=\boldsymbol{\sigma}$, with $\mathbf{S}^{T}=\left(\mathbf{S}_{\mathrm{u}}^{T}\right.$ $\mathbf{S}_{\mathrm{c}}^{T}$ ). In this case, it should be emphasized that the matrix $\mathbf{g}$ in eq. (12) is still nonsingular and its inverse is well defined by $\mathbf{G}$ $\equiv \mathbf{B m}^{-1} \mathbf{B}^{T}$, so long as the whole $\mathbf{S}$ is nonredundant, as we have already assumed. For a given molecular geometry, it is not difficult to find a desired nonredundant set $\mathbf{S}$ of GCs. Thus, applying $\mathbf{G}$ to the left-hand side of eq. (12), we obtain

$$
\begin{aligned}
\ddot{\mathbf{S}}=-\dot{\mathbf{S}}^{T}\left[\mathbf{B} \mathbf{A}_{2}\right] \dot{\mathbf{S}}-\mathbf{B m}^{-1} \partial_{\mathbf{X}} V_{\mathrm{c}} & \\
=\dot{\mathbf{S}}^{T} \mathbf{A}^{T}\left[\mathbf{B}_{2}\right] \mathbf{A} \dot{\mathbf{S}}+\mathbf{B} \ddot{\mathbf{X}}[\text { from eqs. (4) and (8)] } & =\dot{\mathbf{X}}^{T}\left[\mathbf{B}_{2}\right] \dot{\mathbf{X}}+\mathbf{B} \ddot{\mathbf{X}},
\end{aligned}
$$

which is the very expression obtainable from the second derivative of eq. (1) with respect to time. ${ }^{85}$ For the constrained coordinates $\mathbf{S}_{\mathrm{c}}$, this becomes an equation for $\Lambda$ that is equivalent to eq. (11).

\section{Local Energy Drift}

The reliability of long time simulations of a dynamical system depends on the stability of the numerical integration scheme. The local error in total energy in each integration time step eventually affects the global stability, the latter depending on whether the local errors are cumulative or not. To determine the factors that affect the local energy drift, we consider the following general numerical integration scheme in GCs:

$$
\begin{gathered}
\mathbf{S}(\Delta t)=\mathbf{S}(0)+\Delta t \alpha \dot{\mathbf{S}}(0)+\Delta t^{2}\{\beta \ddot{\mathbf{S}}(0)+\gamma \ddot{\mathbf{S}}(\Delta t)\} \\
\dot{\mathbf{S}}(\Delta t)=\dot{\mathbf{S}}(0)+\Delta t\{\varepsilon \ddot{\mathbf{S}}(0)+\omega \ddot{\mathbf{S}}(\Delta t)\}
\end{gathered}
$$

where $\alpha, \beta, \gamma, \varepsilon$, and $\omega$ are appropriate constants and variables $\mathbf{S}, \dot{\mathbf{S}}$, and $\ddot{\mathbf{S}}$ are assumed to satisfy eqs. (9)-(12) at each time step with suitable constraint parameters $\Lambda$. In view of eq. (15), we can expand $\ddot{\mathbf{S}}(\Delta t)$ as

$$
\ddot{\mathbf{S}}(\Delta t)=\ddot{\mathbf{S}}(0)+\Delta t \alpha\left[\left(\dot{\mathbf{S}}^{T} \partial_{\mathbf{S}}\right) \ddot{\mathbf{S}}\right](0)+\cdots,
$$

rewriting eqs. (15) and (16), respectively, by

$$
\begin{aligned}
& \mathbf{S}(\Delta t)=\mathbf{S}(0)+\Delta t \alpha \dot{\mathbf{S}}(0)+\Delta t^{2}(\beta+\gamma) \ddot{\mathbf{S}}(0) \\
&+\Delta t^{3} \alpha \gamma\left[\left(\dot{\mathbf{S}}^{T} \partial_{\mathbf{S}}\right) \ddot{\mathbf{S}}\right](0)+\cdots
\end{aligned}
$$

$\dot{\mathbf{S}}(\Delta t)=\dot{\mathbf{S}}(0)+\Delta t(\varepsilon+\omega) \ddot{\mathbf{S}}(0)$

$$
+\Delta t^{2} \alpha \omega\left[\left(\dot{\mathbf{S}}^{T} \partial_{\mathbf{S}}\right) \ddot{\mathbf{S}}\right](0)+\cdots
$$

Then, the total potential energy of eq. (7) at $\mathbf{S}(\Delta t)$ is Taylor expanded as

$$
\begin{array}{r}
V_{\mathrm{c}}(\Delta t)=V_{\mathrm{c}}(0)+\Delta t \alpha\left[\left(\dot{\mathbf{S}}^{T} \partial_{\mathbf{S}}\right) V_{\mathrm{c}}\right](0)+\frac{\Delta t^{2}}{2}\left[2(\beta+\gamma)\left(\ddot{\mathbf{S}}^{T} \partial_{\mathbf{S}}\right) V_{\mathrm{c}}\right. \\
\left.+\alpha^{2} \dot{\mathbf{S}}^{T}\left(\partial_{\mathbf{S}}^{2} V_{\mathrm{c}}\right) \dot{\mathbf{S}}\right](0)+O\left(\Delta t^{3}\right) .
\end{array}
$$

The matrix $g(\Delta t)$ is also expanded by the same form as this with each $V_{\mathrm{c}}$ being replaced by g. Considering eq. (19), we can expand the kinetic energy of eq. (5) by 


$$
\begin{aligned}
T(\Delta t)= & T(0)+\Delta t \dot{\mathbf{S}}^{T}(0)\left[(\varepsilon+\omega) \mathbf{g} \ddot{\mathbf{S}}+\frac{\alpha}{2}\left\{\left(\dot{\mathbf{S}}^{T} \partial_{\mathbf{S}}\right) \mathbf{g}\right\} \dot{\mathbf{S}}\right](0) \\
& +\frac{\Delta t^{2}}{2}\left[(\varepsilon+\omega)^{2} \ddot{\mathbf{S}}^{T} \mathbf{g S}+2 \alpha \omega \dot{\mathbf{S}}^{T} \mathbf{g}\left\{\left(\dot{\mathbf{S}}^{T} \partial_{\mathbf{S}}\right) \ddot{\mathbf{S}}\right\}\right. \\
& +(\beta+\gamma) \dot{\mathbf{S}}^{T}\left\{\left(\ddot{\mathbf{S}}^{T} \partial_{\mathbf{S}}\right) \mathbf{g}\right\} \dot{\mathbf{S}}+2 \alpha(\varepsilon+\omega) \ddot{\mathbf{S}}^{T}\left\{\left(\dot{\mathbf{S}}^{T} \partial_{\mathbf{S}}\right) \mathbf{g}\right\} \dot{\mathbf{S}} \\
& \left.+\frac{\alpha^{2}}{2} \dot{\mathbf{S}}^{T}\left\{\dot{\mathbf{S}}^{T}\left(\partial_{\mathbf{S}}^{2} \mathbf{g}\right) \dot{\mathbf{S}}\right\} \dot{\mathbf{S}}\right](0)+O\left(\Delta t^{3}\right) .
\end{aligned}
$$

Similarly, the system's total energy, $E \equiv T+V_{\mathrm{c}}$, at $\mathbf{S}(\Delta t)$ can be Taylor expanded by

$$
E(\Delta t)=E(0)+\Delta t E^{(1)}(0)+\frac{\Delta t^{2}}{2} E^{(2)}(0)+\frac{\Delta t^{3}}{6} E^{(3)}(0)+\cdots
$$

By using eqs. (20) and (21), the coefficient $E^{(1)}$ is found to be

$$
E^{(1)}=\dot{\mathbf{S}}^{T}\left\{(\varepsilon+\omega) \mathrm{g} \ddot{\mathbf{S}}+\alpha \partial_{\mathbf{s}} V_{\mathrm{c}}+\frac{\alpha}{2}\left[\left(\dot{\mathbf{S}}^{T} \partial_{\mathbf{S}}\right) \mathbf{g}\right] \dot{\mathbf{S}}\right\}
$$

If $\alpha=\varepsilon+\omega$, this term becomes zero from eqs. (12) and (13). Assuming this and arranging terms of $\Delta t^{2}$ with eq. (12), we obtain

$$
\begin{aligned}
E^{(2)}=\left(2 \beta+2 \gamma-\alpha^{2}\right) \ddot{\mathbf{S}}^{T} \partial_{\mathbf{S}} V_{\mathbf{c}} & +\left(\beta+\gamma+\frac{1}{2} \alpha^{2}\right) \dot{\mathbf{S}}^{T}\left[\left(\ddot{\mathbf{S}}^{T} \partial_{\mathbf{S}}\right) \mathbf{g}\right] \dot{\mathbf{S}} \\
& +\alpha(2 \omega-\alpha) \dot{\mathbf{S}}^{T} \mathbf{g}\left[\left(\dot{\mathbf{S}}^{T} \partial_{\mathbf{S}}\right) \ddot{\mathbf{S}}\right]
\end{aligned}
$$

It is evident that there are no nonzero real values for $\beta+\gamma$ such that $2(\beta+\gamma)-\alpha^{2}=0$ and $2(\beta+\gamma)+\alpha^{2}=0$. If we choose $\alpha=\varepsilon+\omega=2 \omega$ and $2(\beta+\gamma)=\alpha^{2}$, which is one of the best possibilities of reducing absolute magnitudes of $E^{(2)}$ including the velocity Verlet ${ }^{88}$ equivalent $(\alpha=1, \beta=\varepsilon=\omega=$ $1 / 2$, and $\gamma=0$ ) in GCs, then the $E(\Delta \mathrm{t})$ is found to be

$$
E(\Delta t)=E(0)+\frac{\Delta t^{2}}{2} \alpha^{2}\left\{\dot{\mathbf{S}}^{T}\left[\left(\ddot{\mathbf{S}}^{T} \partial_{\mathbf{S}}\right) \mathbf{g}\right] \dot{\mathbf{S}}\right\}(0)+O\left(\Delta t^{3}\right)
$$

Different from this, our recent analysis for the corresponding CCMD scheme showed that in terms of CCs the total energy is expanded by ${ }^{84}$

$$
\begin{aligned}
E(\Delta t)=E(0)+ & \frac{\Delta t^{3}}{12}\left\{3 \alpha\left(4 \gamma+\alpha^{2}\right) \ddot{\mathbf{X}}^{T}\left(\partial_{\mathbf{X}}^{2} V_{\mathrm{c}}\right) \dot{\mathbf{X}}\right. \\
& \left.-\alpha^{3} \dot{\mathbf{X}}^{T}\left[\left(\dot{\mathbf{X}}^{T} \partial_{\mathbf{X}}\right)\left(\partial_{\mathbf{X}}^{2} V_{\mathrm{c}}\right)\right] \dot{\mathbf{X}}\right\}(0)+O\left(\Delta t^{4}\right)
\end{aligned}
$$

Therefore, it is clear that, when $\partial_{\mathbf{s}} \mathbf{g} \neq \mathbf{0}$, the general ICMD scheme generates a local error in energy during each time step that is one-order larger in $\Delta t$ than that resulting from the CCMD scheme. The $\Delta t^{2}$-dependence of the leading local error in terms of GCs as given by eq. (25) is consistent with the result in terms of the Riemannian coordinates of Gibson and Scheraga. ${ }^{52}$ Note that if $\mathbf{S}=\mathbf{X}$ then $\partial_{\mathbf{S}} \mathbf{g}=\partial_{\mathbf{X}} \mathbf{m}=\mathbf{0}$ resulting in $E^{(2)}=0$. Thus, the very term $\partial_{\mathbf{s}} \mathbf{g}$, which causes the equations of motion to be nonlinear if it is nonzero, enforces the dynamics integration scheme in GCs to be less stable than that in CCs. This theoretical analysis is consistent with the simulation results for a system of an isolated octane molecule, the details being presented in Application to an $n$-Octane Molecule.

\section{ICMD Algorithms}

Dynamics trajectory information in GCs requires computing $\ddot{\mathbf{S}}$ at each integration time step. As shown in eq. (14), this involves computing either $\mathbf{A}_{2}$ or $\mathbf{B}_{2}$. Since calculation of $\mathbf{A}_{2}$ is nontrivial and requires larger storage than that of $\mathbf{B}_{2}$, we have investigated dynamics processes using $\mathbf{B}_{2}$. In the presence of the internal constraints of eq. (6), however, we additionally need to determine $\Lambda$ either by solving eq. (11) or in another way. We label any unconstrained and constrained quantities in GCs by " $u$ " and "c", respectively. Two ICMD schemes further refined from our early B-matrix ICMD method ${ }^{82,83}$ are presented later.

\section{Algorithm AICMD}

This integration scheme updates positions and velocities in GCs. Since, in the presence of internal constraints, we can determine values of $\dot{\mathbf{S}}_{\mathrm{u}}(\Delta t)$ only after knowing values of $\ddot{\mathbf{S}}(\Delta t)$ [see eq. (16)], we initially approximate $\dot{\mathbf{S}}_{\mathrm{u}}(\Delta t)$ by

$$
\dot{\mathbf{R}}_{\mathrm{u}}(\Delta t)=\dot{\mathbf{S}}_{\mathrm{u}}(0)+\Delta t \ddot{\mathbf{S}}_{\mathrm{u}}(0) .
$$

From this, $\dot{\mathbf{S}}_{\mathrm{u}}(\Delta t)$ and $\ddot{\mathbf{S}}_{\mathrm{u}}(\Delta t)$ are iteratively adjusted to follow the velocity Verlet equivalent with

$$
\dot{\mathbf{w}}_{\mathrm{u}}(\Delta t) \equiv \dot{\mathbf{S}}_{\mathrm{u}}(0)+\frac{\Delta t}{2} \ddot{\mathbf{S}}_{\mathrm{u}}(0)
$$

The AICMD procedures are summarized as follows:

a. Compute $\mathbf{A}(k), \mathbf{B}(k), \mathbf{B}_{2}(k)$, and $\mathbf{F}_{X}(k) \equiv-\partial_{\mathbf{X}(k)} V$.

b. Set initial atomic velocities: $\dot{\mathbf{X}}(k) \equiv \mathbf{A}_{\mathrm{u}}(k) \dot{\mathbf{R}}_{\mathrm{u}}(k)$.

c. Iterate for $\dot{\mathbf{S}}_{\mathrm{u}}(k)$ and $\ddot{\mathbf{S}}_{\mathrm{u}}(k)$ :

1. Solve $\mathbf{G}_{\mathrm{cc}} \Lambda(k)=\mathbf{B}_{\mathrm{c}}(k) \mathbf{m}^{-1} \mathbf{F}_{X}(k)+\dot{\mathbf{X}}^{T}(k) \mathbf{B}_{2 \mathrm{c}}(k) \dot{\mathbf{X}}(k)$ for $\Lambda(k)$.

2. Obtain $\mathrm{CC}$ accelerations: $\ddot{\mathbf{X}}(k)=\mathbf{m}^{-1} \mathbf{F}_{X}(k)-\mathbf{m}^{-1} \mathbf{B}_{\mathrm{c}}^{T}(k) \Lambda(k)$.

3. Obtain GC accelerations: $\ddot{\mathbf{S}}_{\mathrm{u}}(k)=\mathbf{B}_{\mathrm{u}} \ddot{\mathbf{X}}(k)+\dot{\mathbf{X}}^{T}(k) \mathbf{B}_{2 \mathrm{u}}(k) \dot{\mathbf{X}}(k)$.

4. Set $\dot{\mathbf{S}}_{\mathrm{u}}(k)=\dot{\mathbf{w}}_{\mathrm{u}}(k)+\Delta t \ddot{\mathbf{S}}_{\mathrm{u}}(k) / 2$ [velocity Verlet equivalent in GCs].

5. Set $\dot{\mathbf{X}}(k)=\mathbf{A}_{\mathrm{u}}(k) \dot{\mathbf{S}}_{\mathrm{u}}(k)$ and go to (1).

d. Set $\dot{\mathbf{w}}_{\mathrm{u}}(k+1) \equiv \dot{\mathbf{S}}_{\mathrm{u}}(k)+\Delta t \ddot{\mathbf{S}}_{\mathrm{u}}(k) / 2$ and $\dot{\mathbf{R}}_{\mathrm{u}}(k+1) \equiv \dot{\mathbf{S}}_{\mathrm{u}}(k)$ $+\Delta t \ddot{\mathbf{S}}_{\mathrm{u}}(k)$.

e. Set new GC values: $\mathbf{S}_{\mathrm{u}}(k+1)=\mathbf{S}_{\mathrm{u}}(k)+\Delta t \dot{\mathbf{w}}_{\mathrm{u}}(k+1)$.

f. Compute $\mathbf{X}(k+1)$ from $\mathbf{S}(k+1)$.

g. Go to (a) with $k=k+1$. 


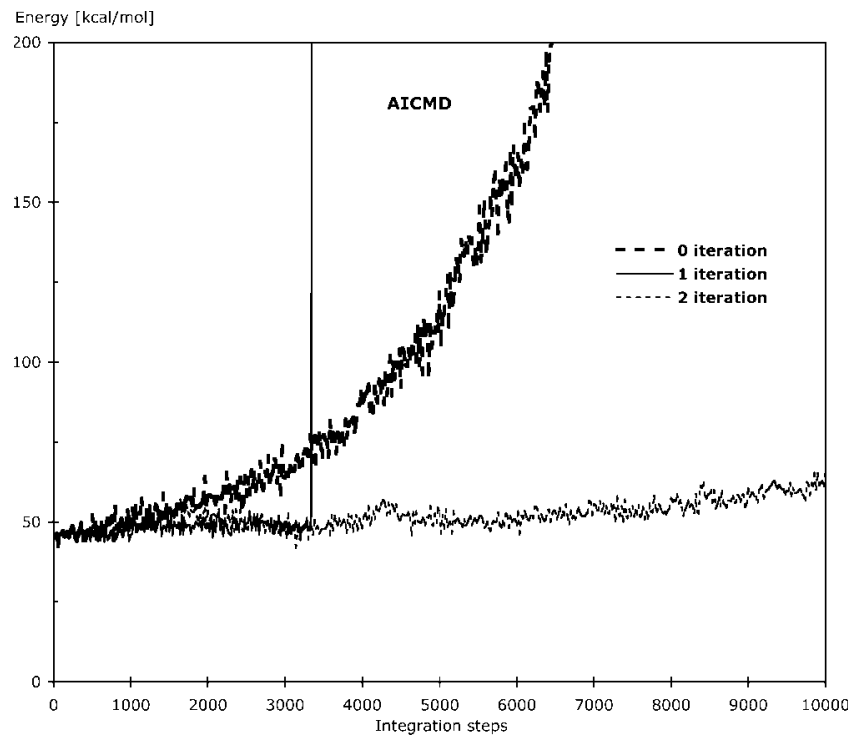

Figure 1. Energy $(T+V)$ versus integration steps for constrained AICMD simulations with $\Delta t=2 \mathrm{fs}$, and 0,1 , and 2 iterations of GC velocities [see AICMD scheme]. The 1-iteration curve ends with the spike.

For given atomic velocities in GCs, the constrained atomic velocities in CCs are obtained by step (b). The computation cost involved in the iteration of step (c) is negligible compared to that of calculating $\mathbf{F}_{X}$. With zero iteration meaning no return from (c5) to step (c1), in most cases two iterations are enough to reach a convergence in $\dot{\mathbf{S}}_{\mathrm{u}}$ that maintains stable energies (Fig. 1).

When there is no constraint, we skip the linear equation solving of (c1) and atomic accelerations are simply given by $\ddot{\mathbf{X}}=$ $\mathbf{m}^{-1} \mathbf{F}_{X}$.

\section{Algorithm BICMD}

When GC accelerations are determined by the last expression of eq. (14), computing $\dot{\mathbf{X}}$ and $\ddot{\mathbf{X}}$ are required at each time step. Thus, in this scheme, atomic velocities are updated in CCs and positions are updated in GCs at each integration time step. ${ }^{85}$ With internal constraints of eq. (6), the desired atomic velocities and accelerations in $\mathrm{CCs}$ can be efficiently determined in the same way as in WIGGLE. ${ }^{84}$ By defining

$$
\dot{\mathbf{q}}(\Delta t) \equiv \frac{1}{\Delta t}\{\mathbf{X}(\Delta t)-\mathbf{X}(0)\}
$$

the BICMD scheme is specifically outlined as follows:

a. Compute $\mathbf{B}(k), \mathbf{B}_{2}(k)$, and $\mathbf{F}_{X}(k) \equiv-\partial_{\mathbf{X}(k)} V$.

b. Set initial atomic velocities: $\dot{\mathbf{Z}}(k)=\dot{\mathbf{q}}(k)+\Delta t \mathbf{m}^{-1} \mathbf{F}_{X}(k) / 2$.

c. Adjust $\dot{\mathbf{X}}(k)$ from the $\dot{\mathbf{Z}}(k)$ so that $\mathbf{B}_{c}(k) \dot{\mathbf{X}}(k)=\mathbf{0}$ :

1. Solve $\mathbf{G}_{\mathrm{cc}} \Gamma\left(k=\mathbf{B}_{\mathrm{c}}(k) \Gamma(k)\right.$ for $\Gamma(k)$.

2. Compute desired velocities: $\dot{\mathbf{X}}(k)=\dot{\mathbf{Z}}(k)-\mathbf{m}^{-1} \mathbf{B}_{\mathrm{c}}^{T}(k) \Gamma(k)$.

d. Set CC accelerations: $\ddot{\mathbf{X}}(k)=2\{\dot{\mathbf{X}}(k)-\dot{\mathbf{q}}(k)\} / \Delta t$ [velocity Verlet].

e. Obtain $\dot{\mathbf{S}}_{\mathrm{u}}(k)=\mathbf{B}_{\mathrm{u}} \dot{\mathbf{X}}(k)$ and $\ddot{\mathbf{S}}_{\mathrm{u}}(k)=\mathbf{B}_{\mathrm{u}} \ddot{\mathbf{X}}(k)+\dot{\mathbf{X}}^{T}(k) \mathbf{B}_{2 \mathrm{u}} \dot{\mathbf{X}}(k)$. f. Set new GC values: $\mathbf{S}_{\mathbf{u}}(k+1)=\mathbf{S}_{\mathrm{u}}(k)+\Delta t\left\{\dot{\mathbf{S}}_{\mathrm{u}}(k)+\Delta t \ddot{\mathbf{S}}_{\mathrm{u}}(k) /\right.$ $2\}$.

g. Compute $\mathbf{X}(k+1)$ from $\mathbf{S}(k+1)$

h. Set $\dot{\mathbf{q}}(k+1)=\frac{1}{\Delta t}\{\mathbf{X}(k+1)-\mathbf{X}(k)\}$.

i. Go to (a) with $k=k+1$.

At each time step, the above process requires adjusting $\dot{\mathbf{X}}$ so as to satisfy the hidden constraints [eq. (9)], which can be expedited by the WIGGLE method. ${ }^{84}$ For more accurate $\ddot{\mathbf{X}}(k)$ with an additional linear equation solving, step (d) can be replaced by d. Obtain atomic accelerations:

1. Solve $\mathbf{G}_{\mathrm{cc}} \Lambda(k)=\mathbf{B}_{\mathrm{c}}(k) \mathbf{m}^{-1} \mathbf{F}_{X}(k)+\dot{\mathbf{X}}^{T}(k) \mathbf{B}_{2 \mathrm{c}}(k) \dot{\mathbf{X}}(k)$ for $\Lambda(k)$.

2. Compute $\mathrm{CC}$ accelerations: $\ddot{\mathbf{X}}(k)=\mathbf{m}^{-1} \mathbf{F}_{X}(k)-\mathbf{m}^{-1} \mathbf{B}_{\mathrm{c}}^{T}(k) \Lambda(k)$.

For the corresponding unconstrained dynamics scheme, steps (c), (d), and (h) are replaced by

$$
\begin{gathered}
\dot{\mathbf{X}}(k)=\dot{\mathbf{q}}(k)+\frac{\Delta t}{2} \ddot{\mathbf{X}}(k) \\
\dot{\mathbf{q}}(k+1)=\dot{\mathbf{X}}(k)+\frac{\Delta t}{2} \ddot{\mathbf{X}}(k),
\end{gathered}
$$

with $\ddot{\mathbf{X}}(k)=\mathbf{m}^{-1} \mathbf{F}_{X}(k)$.

Our test of an isolated octane molecule shows that the constrained BICMD scheme slowly dissipates the system's total energy even with a small time step (Fig. 2). Such a decrease in energy disappears for unconstrained BICMD simulations.

\section{Application to an $n$-Octane Molecule}

For comparisons of dynamic characteristics between CCMD and ICMD simulations, we consider a system of an isolated $n$-octane

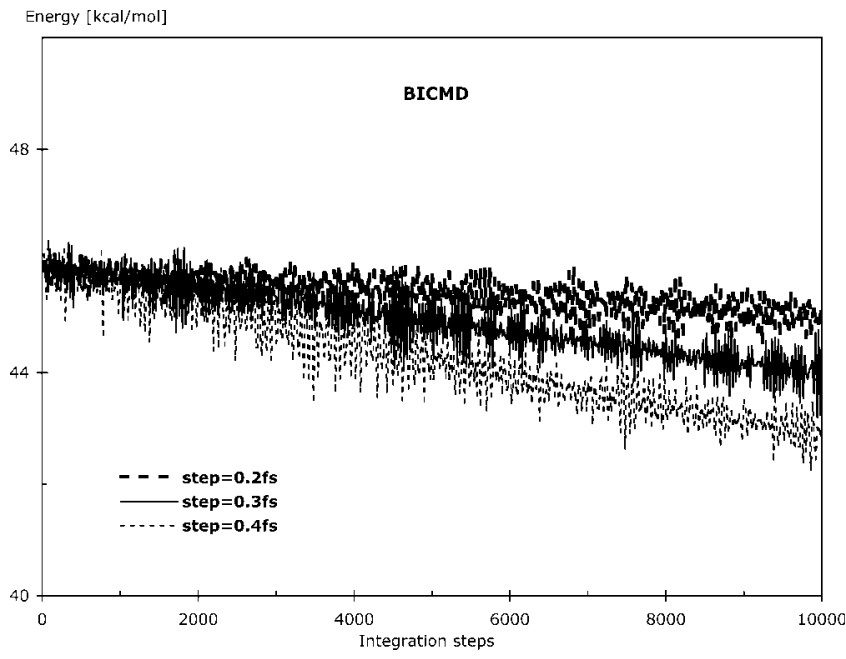

Figure 2. Energy $(T+V)$ versus integration steps for constrained BICMD simulations with different $\Delta t$. 
molecule. Since some $\mathrm{C}-\mathrm{C}$ stretching frequencies are within the range of some angle bending frequencies, we fix only the $\mathrm{CH}$ bond lengths (to $1.08 \AA$ ) for constrained simulations. All the linear equations involved in these constrained dynamics simulations were solved by the conjugate gradient method. Specifically, the method was preconditioned by diagonal elements of $\mathbf{G}_{\mathrm{cc}}$ with a tolerance of $10^{-8}$ for the weighted square of the residual. ${ }^{90}$ The constrained CCMD algorithms used for the simulation results labeled SHAKE and RATTLE differ from their original versions. Details of these modifications of the original SHAKE ${ }^{39}$ and RAT$\mathrm{TLE}^{40}$ algorithms were reported in our previous paper. ${ }^{84}$

Computations were done on a single node (512 Mbytes of memory and $1.2 \mathrm{GHz}$ processing speed) of a LINUX cluster, using the SDFF force field for hydrocarbon chains optimized to reproduce $a b$ initio structures, energies, and vibrational frequencies. ${ }^{91}$ The system was equilibrated to provide average values of temperature and energy $(T+V)$ (for 1000 steps with $\Delta t=0.5 \mathrm{fs}$ ) of $300.2 \mathrm{~K}$ and $45.86 \mathrm{kcal} / \mathrm{mol}$, respectively, for constrained simulations $(300 \mathrm{~K}$ and $42.20 \mathrm{kcal} / \mathrm{mol}$ for unconstrained simulations). The desired initial configuration had atomic velocities correspond-
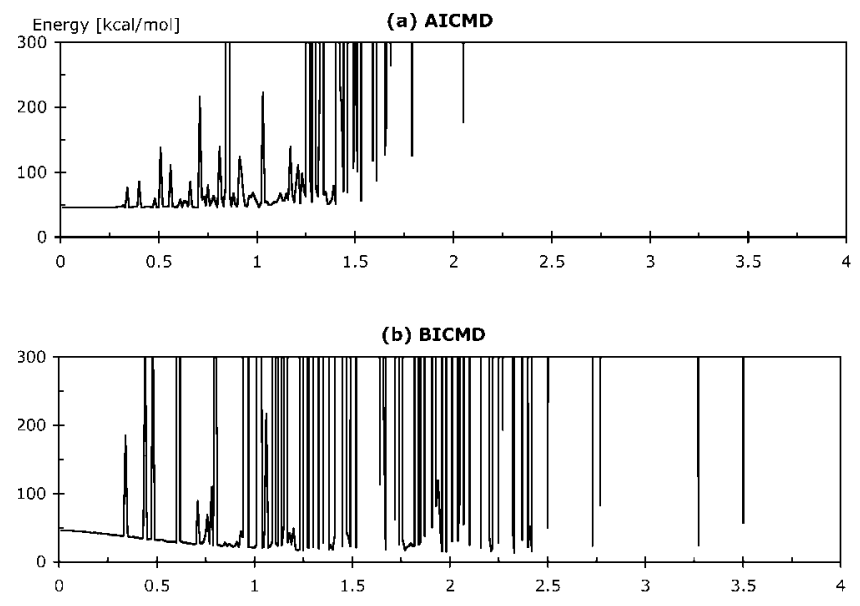

(c) WIGGLE

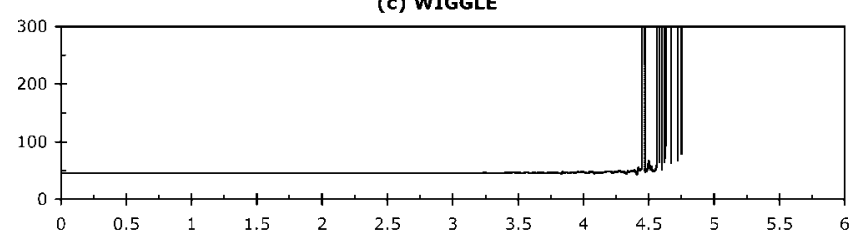

(d) SHAKE

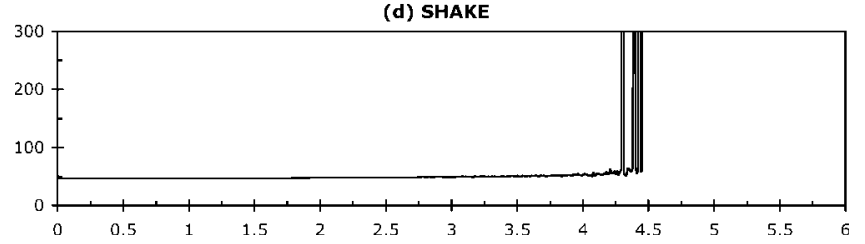

Figure 3. Energy $[\mathrm{kcal} / \mathrm{mol}]$ versus time step $\Delta t[\mathrm{fs}]$ for various constrained $\mathrm{MD}$ simulations of an isolated $n$-octane molecule: (a) AICMD, (b) BICMD, (c) WIGGLE, and (d) SHAKE. Instantaneous energies $(T+V)$ at the 50,000th integration step are shown. The time step was scanned in increments of $0.01 \mathrm{fs}$.

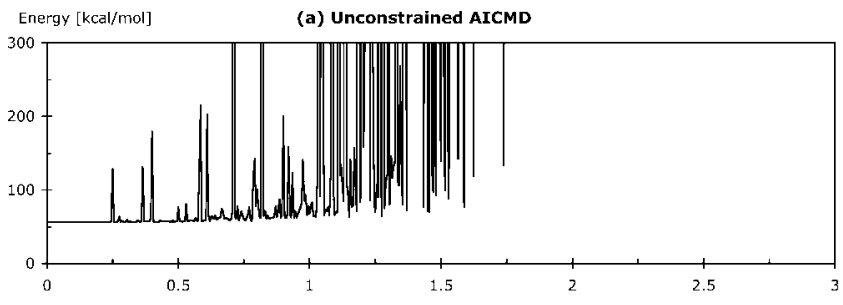

(b) Unconstrained BICMD

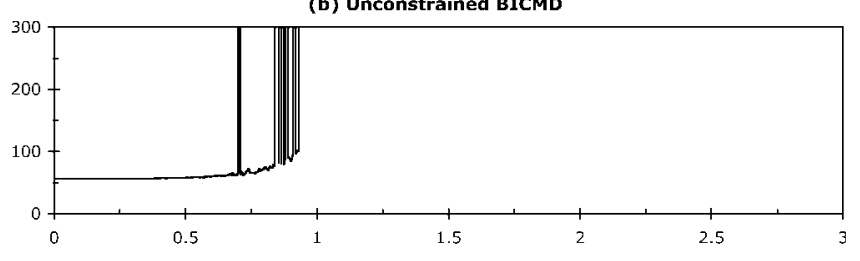

(c) Unconstrained CCMD

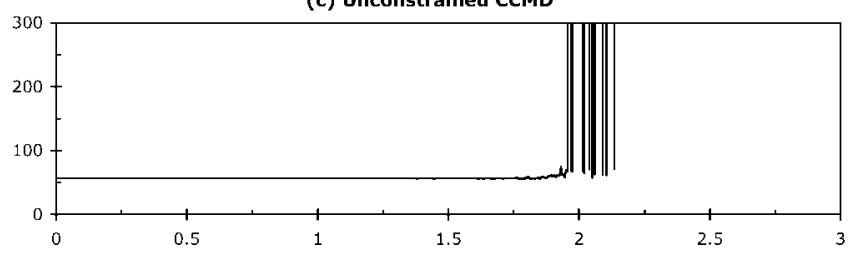

Figure 4. Total energy $[\mathrm{kcal} / \mathrm{mol}]$ versus time step $\Delta t[\mathrm{fs}]$ for unconstrained MD simulations: (a) AICMD, (b) BICMD, and (c) velocity Verlet CCMD. Instantaneous energies at the 50,000th integration step are shown. The time step was scanned in increments of $0.005 \mathrm{fs}$.

ing to an instantaneous temperature of $312.9 \mathrm{~K}$ for constrained simulations (350.2 $\mathrm{K}$ for unconstrained simulations), with all backbone $\tau(\mathrm{C}-\mathrm{C}-\mathrm{C}-\mathrm{C})$ torsions having trans conformations except for the center torsion angle of $52^{\circ}$ for both constrained and unconstrained MD simulations. The initial atomic velocities were adjusted so as to remove linear and angular momenta about the center of mass by using our previous method. ${ }^{84}$

Although the system's total energy contains potential energy $\boldsymbol{\sigma}^{T} \Lambda$ due to constraint forces for internal constraints, this is ignored in our figures. Stability of constrained MD simulations can also be tested by monitoring values of $T+V$. Figure 3 shows instantaneous energy $(T+V$ in $\mathrm{kcal} / \mathrm{mol}$ ) versus time step $\Delta t$ (in fs) at the 50,000th integration step for different methods of constrained ICMD and CCMD simulations with internal constraints on all $\mathrm{CH}$ bond lengths. Results from unconstrained MD simulations are shown in Figure 4. It is seen that MD simulations in CCs are more stable than those in ICs, consistent with the theoretical analysis presented in Local Energy Drift. In ICMD simulations, stable regions in $\Delta t$ can be extended toward an increased time step by imposing rigid constraints on all $\mathrm{CH}$ bond lengths, but ripples in energy, which are a direct indication of the system's instability, still exist even below $0.5 \mathrm{fs}$. The ripples in the region of a fairly small $\Delta t$, where the corresponding constrained CCMD simulations are stable, seem to arise from the nonlinearity of the equations of motion in ICs in contrast to the linearity in CCs. Constrained MD simulation results at the 100,000th integration step are shown in Figure 5. This also shows that CCMD sim- 


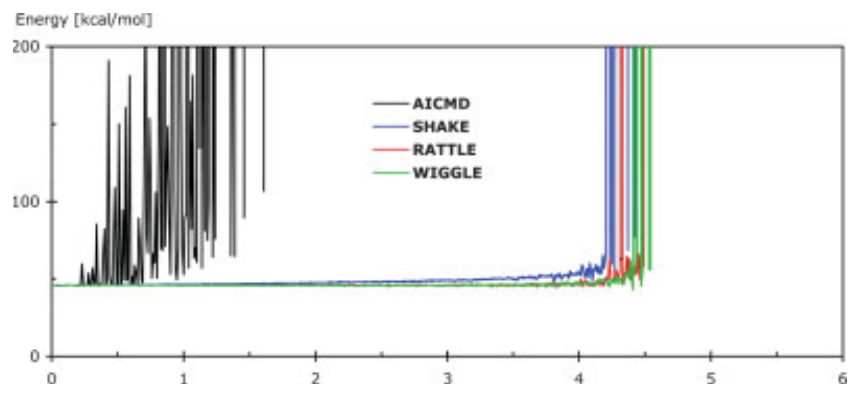

Figure 5. Energy $[\mathrm{kcal} / \mathrm{mol}]$ versus time step $\Delta t[\mathrm{fs}]$ for various constrained MD simulations of an isolated $n$-octane molecule: AICMD (black), SHAKE (blue), RATTLE (red), and WIGGLE (green). Instantaneous energies $(T+V)$ at the 100,000th integration step are shown. The time step was scanned in increments of $0.01 \mathrm{fs}$.

ulations are more stable than ICMD simulations, with the WIGGLE scheme being slightly better in stability than the revised SHAKE and RATTLE for constrained CCMD simulations. We have used two iteration cycles for the iteration step (c) for AICMD simulation results shown in Figures 4 and 5.

\section{Concluding Remarks}

Early MD methodology in ICs was developed for efficient analyses of conformation energy of polypeptides and proteins, and was based on torsion angles. Torsion angle MD simulations have been especially efficient in structure determinations of biomolecules by NMR spectroscopy and X-ray crystallography. Extending this work, some reports ${ }^{52,68,72,73}$ of stable MD simulations in torsion angles with integration time steps greater than $4 \mathrm{fs}$ encouraged further improvements in ICMD methodology. This was supported by detailed studies by Mazur, ${ }^{60,86}$ which included all (bond length, bond angle, and torsion) ICs and concluded that there were no essential differences between ICMD and CCMD simulations. However, the situation has remained controversial, with Stocker et al, stating that “..., for equilibrium simulations, Newton's equations of motion in Cartesian coordinates are to be preferred to Lagrange's equations of motion using generalized, non-Cartesian, coordinates." 87

We have made an intensive comparative study of ICMD and CCMD simulations, and obtained several results. First, for a general numerical integration scheme for ICMD simulations, we have investigated an analytic relation that provides local error in energy during an integration time step $\Delta t$. The leading local error term is found to be proportional to $\Delta t^{2}$ and to contain $\partial_{\mathrm{S}} \mathbf{g}$, viz., the first derivative of the mass-matrix $\mathbf{g}$ with respect to GCs [see eq. (25)]. This is an order in integration step larger than that proportional to $\Delta t^{3}$ for the corresponding CCMD scheme. ${ }^{84}$ Therefore, the term $\partial_{\mathrm{S}} \mathbf{g} \neq \mathbf{0}$ itself, which causes the related equations of motion to be nonlinear in GCs, is found to make ICMD simulations less stable than CCMD simulations. Second, we have introduced two ICMD schemes, AICMD and BICMD, that incorporate the second order B-matrix elements instead of the second order A-matrix elements. In the AICMD scheme, GC velocities and accelerations are iteratively adjusted so as to conform to the velocity Verlet equivalent in GCs. In the BICMD scheme, atomic velocities with internal constraints are updated and adjusted in CCs based on the WIGGLE constrained CCMD scheme. ${ }^{84}$ Finally, the proposed ICMD schemes have been applied to an isolated octane molecule and their performances compared with several CCMD schemes. The resulting analyses clearly show that ICMD simulations are less stable than CCMD simulations, with/without rigid constraints on all $\mathrm{CH}$ bond lengths. By constraining all $\mathrm{CH}$ bond lengths, some increased integration time steps can be found for stable ICMD simulations, but there are also some regions in $\Delta t$ even smaller than 0.5 fs that generate unstable trajectories.

As far as stability is concerned, for pure MD simulations $\mathrm{CCs}$ are preferred to ICs, although ICs are efficient in $a b$ initio geometry optimizations, MC simulations, and structural refinements for biomolecules. Any needed trajectory information in ICs can easily be obtained from that of CCMD simulations with calculated nonzero elements of B-matrices [see eqs. (1) and (14)]. As a final note, we would like to mention that dynamic trajectories are believed to be more stable for symplectic integrators than those for nonsymplectic ones in long time simulations. RATTLE has proved to be symplectic while SHAKE is not. ${ }^{92}$ We think it is nontrivial and beyond the scope of the present article to determine whether our presented AICMD and BICMD algorithms are symplectic or not. However, we have shown that dynamical instability in ICMD simulations will arise in any case from the nonlinearity in the equations of motion.

\section{Acknowledgments}

The authors thank Dr. Robert Krasny for many helpful discussions.

\section{Appendix}

In deriving eq. (14) from eq. (12), we have used eqs. (3) and (4), viz., the nonsingularity relations between CCs $\mathbf{X}$ and GCs $\mathbf{S}$. Desired nonsingular transformation matrices $\mathbf{B}, \mathbf{B}_{2}, \mathbf{A}$, and $\mathbf{A}_{2}$ can always be determined for a set $\mathbf{S}$ of nonredundant GCs that is suitably chosen by combining nonredundant ICs with external rotations and translations. The calculation of nonzero elements of $\mathbf{B}$ and $\mathbf{B}_{\mathbf{2}}$ for external rotations has conventionally been accomplished $^{1,2,93}$ by assuming the Casimir-Eckart conditions. ${ }^{94-96}$ Previously, we developed an efficient method of computing these without imposing the Casimir-Eckart conditions. ${ }^{83}$ We present here a further improvement in this method.

\section{External Rotations with Three Parameters}

To compute the B-matrix elements for external rotations, we need to define a suitable molecule-fixed (MF) coordinate frame and its transformation (viz., rotation matrix) to an arbitrary laboratoryfixed (LF) coordinate frame in terms of the relative rotation angles $\vec{\phi}=\left(\phi^{1} \phi^{2} \phi^{3}\right)^{T}$ between the two frames. Let $\left\{\hat{\mathbf{1}}_{\mathrm{MF}}, \hat{\mathbf{2}}_{\mathrm{MF}}, \hat{\mathbf{3}}_{\mathrm{MF}}\right\}$ be an arbitrary orthonormal basis for the MF frame with $\hat{\mathbf{3}}_{\mathrm{MF}}=\hat{\mathbf{1}}_{\mathrm{MF}} \times \hat{\mathbf{2}}_{\mathrm{MF}}$ and any quantity in this frame being labeled by "MF." Let $\{\hat{\mathbf{1}}, \hat{\mathbf{2}}, \hat{\mathbf{3}}\}$ be the standard basis for the LF (CC) frame with $\hat{\mathbf{1}} \equiv\left(\begin{array}{lll}1 & 0 & 0\end{array}\right)^{T}, \hat{\mathbf{2}} \equiv\left(\begin{array}{lll}0 & 1 & 0\end{array}\right)^{T}$, and $\hat{\mathbf{3}} \equiv\left(\begin{array}{lll}0 & 0 & 1\end{array}\right)^{T}$. The 
position of a molecule's center-of-mass is determined only after all atomic positions are known. To expedite ICMD simulations for flexible molecules, instead of the conventional center-of-mass frame, we adopt an MF frame whose origin is located at a particular atom center $\mathbf{x}_{\zeta}$. A basis for the MF frame can easily be found from any three nonlinear atomic positions including the origin atom $\zeta$. Without a loss of generality, we define the transformation matrix between the two frames by. ${ }^{93}$

$$
\begin{aligned}
& \Xi(\vec{\phi}) \equiv \exp \left(\phi^{3} \mathbf{D}_{3}\right) \exp \left(\phi^{2} \mathbf{D}_{2}\right) \exp \left(\phi^{1} \mathbf{D}_{1}\right) \\
& =\left(\begin{array}{ccc}
c_{3} c_{2} & c_{3} s_{2} s_{1}-s_{3} c_{1} & c_{3} s_{2} c_{1}+s_{3} s_{1} \\
s_{3} c_{2} & s_{3} s_{2} s_{1}+c_{3} c_{1} & s_{3} s_{2} c_{1}-c_{3} s_{1} \\
-s_{2} & c_{2} s_{1} & c_{2} c_{1}
\end{array}\right)
\end{aligned}
$$

with $c_{j} \equiv \cos \phi^{j}, s_{j} \equiv \sin \phi^{j}$, and

$$
\begin{aligned}
\mathbf{D}_{1} \equiv\left(\begin{array}{ccc}
0 & 0 & 0 \\
0 & 0 & -1 \\
0 & 1 & 0
\end{array}\right), \mathbf{D}_{2} \equiv\left(\begin{array}{ccc}
0 & 0 & 1 \\
0 & 0 & 0 \\
-1 & 0 & 0
\end{array}\right), \\
\mathbf{D}_{3} \equiv\left(\begin{array}{ccc}
0 & -1 & 0 \\
1 & 0 & 0 \\
0 & 0 & 0
\end{array}\right) .
\end{aligned}
$$

Then, atomic coordinates in the LF frame are related to that in MF frame by

$$
\mathbf{x}_{\lambda}=\Xi(\vec{\phi}) \mathbf{x}_{\lambda \mathrm{MF}}+\mathbf{x}_{\zeta}
$$

To be more specific in defining a basis for the MF frame, let atom $i_{1}$, atom $i_{2}$, and atom $i_{3}$ be such nonlinear atoms as shown in Figure 6, with one of these being the origin of the MF frame. Defining $\mathbf{x}_{21} \equiv \mathbf{x}_{i_{1}}-\mathbf{x}_{i_{2}}, \mathbf{x}_{23} \equiv \mathbf{x}_{i_{3}}-\mathbf{x}_{i_{2}}, \boldsymbol{r}_{21} \equiv\left|\mathbf{x}_{21}\right|, \boldsymbol{r}_{23} \equiv$ $\left|\mathbf{x}_{23}\right|, \mathbf{e}_{21} \equiv \mathbf{x}_{21} / \boldsymbol{r}_{21}, \mathbf{e}_{23} \equiv \mathbf{x}_{23} / \boldsymbol{r}_{23}$, and $\cos \varphi_{13} \equiv \mathbf{e}_{21} \cdot \mathbf{e}_{23}$, we obtain an orthonormal vector set $\left\{\mathbf{e}_{21}, \mathbf{u} \equiv \mathbf{e}_{21} \times \mathbf{e}_{23} / \sin \varphi_{13}\right.$, $\left.\mathbf{v} \equiv \mathbf{u} \times \mathbf{e}_{21}\right\}$ in the LF frame. In view of eq. (A3), the corresponding vectors in the MF frame should satisfy

$$
\left\{\mathbf{e}_{21}, \mathbf{v}, \mathbf{u}\right\}=\Xi(\vec{\phi})\left\{\mathbf{e}_{21 \mathrm{MF}}, \mathbf{v}_{\mathrm{MF}}, \mathbf{u}_{\mathrm{MF}}\right\}
$$

Then a desired orthonormal basis for the MF frame can be chosen so as to satisfy

$$
\begin{aligned}
& \mathbf{e}_{21 \mathrm{MF}} \equiv n_{1} \hat{\mathbf{1}}_{\mathrm{MF}}=\left(\begin{array}{lll}
n_{1} & 0 & 0
\end{array}\right)_{\mathrm{MF}}^{T} \\
& \mathbf{v}_{\mathrm{MF}} \equiv n_{2} \hat{\mathbf{z}}_{\mathrm{MF}}=\left(\begin{array}{lll}
0 & n_{2} & 0
\end{array}\right)_{\mathrm{MF}}^{T} \\
& \mathbf{u}_{\mathrm{MF}} \equiv n_{3} \hat{\mathbf{3}}_{\mathrm{MF}}=\left(\begin{array}{lll}
0 & 0 & n_{3}
\end{array}\right)_{\mathrm{MF}}^{T}
\end{aligned}
$$

with

$$
\begin{gathered}
n_{1} n_{1}=1=n_{2} n_{2}=n_{3} n_{3} \\
n_{1} n_{2} n_{3}=1 .
\end{gathered}
$$

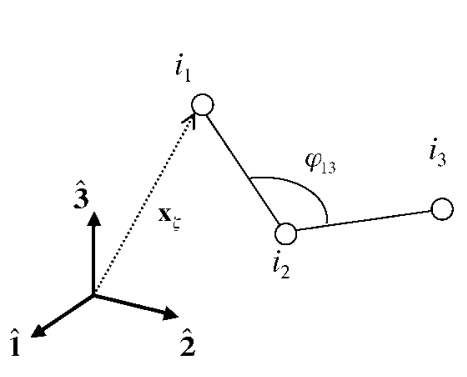

(a)

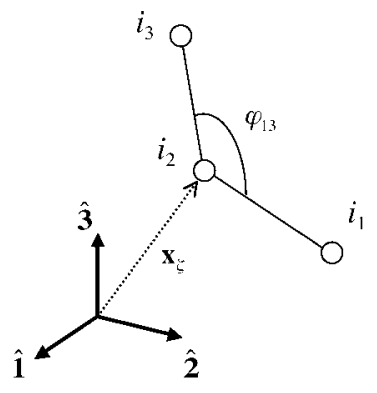

(b)
Figure 6. Definition of molecule fixed (MF) coordinate frames with three nonlinear atoms containing the origin atom $\zeta$ : (a) for $\left(n_{1}, n_{2}\right.$, $\left.n_{3}\right)=(-1,1,-1)$ and $(\mathrm{b})$ for $\left(n_{1}, n_{2}, n_{3}\right)=(1,1,1)$.

Relations (A8) and (A9) are simply conditions for normalization and a proper orientation, respectively. When a suitable MF frame is specifically chosen, the values of $\left(n_{1}, n_{2}, n_{3}\right)$ are also fixed. For example, these can be taken as $(-1,1,-1)$ and $(1,1$, 1) for the cases of Figure $6 a$ and $6 b$, respectively. For our simulation results in Application to an $n$-Octane Molecule, we have used an MF frame as Figure 6a. Physical properties derived from MD trajectories are independent on the choice of an MF frame. By substituting eqs. (A5)-(A7) into (A4), equations for $\vec{\phi}$ are found to be

$$
\begin{gathered}
s_{2}=-n_{1} e_{21}^{3} \\
c_{1}=n_{3} u^{3} / c_{2}, \quad s_{1}=n_{2} \nu^{3} / c_{2} \\
c_{3}=n_{1} e_{21}^{1} / c_{2}, \quad s_{3}=n_{1} e_{21}^{2} / c_{2} .
\end{gathered}
$$

Thus, from eq. (A10) $\phi^{2}$ can be first determined within the range of $-\pi / 2<\phi^{2}<\pi / 2$, and next $\phi^{1}$ and $\phi^{3}$ are determined from eqs. (A11) and (A12), respectively. The corresponding B-matrix elements, viz., $\phi_{\lambda^{k}}^{l} \equiv \partial \phi^{l} / \partial x_{\lambda}^{k}$ or $\phi_{\nu j \lambda^{k}}^{l} \equiv \partial^{2} \phi^{l} / \partial x_{\nu}^{j} \partial x_{\lambda}^{k}$, can be computed by directly differentiating eqs. (A10)-(A12) with respect to atomic coordinates $\mathbf{x}_{\lambda}$ or $\mathbf{x}_{\nu}$, which are nonzero at most for $\lambda, \nu=i_{1}, i_{2}$, and $i_{3}$.

Another direct way of computing B-matrix elements for external rotation can be found from the first derivative of eq. (A4) with respect to atomic coordinates ${ }^{83}$ :

$$
\left\{\frac{\partial \mathbf{e}_{12}}{\partial x_{\lambda}^{k}}, \frac{\partial \mathbf{v}}{\partial x_{\lambda}^{k}}, \frac{\partial \mathbf{u}}{\partial x_{\lambda}^{k}}\right\}=\vec{\theta}_{\lambda^{k}} \times\left\{\mathbf{e}_{12}, \mathbf{v}, \mathbf{u}\right\}
$$

with $\vec{\theta}_{\lambda^{k}} \equiv \partial \vec{\theta} / \partial x_{\lambda}^{k}$ being related to $\phi_{\lambda^{k}}^{l}$ by the transformation of

$$
\left(\begin{array}{c}
\theta_{\lambda^{k}}^{1} \\
\theta_{\lambda^{k}}^{2} \\
\theta_{\lambda^{k}}^{3}
\end{array}\right) \equiv\left(\begin{array}{ccc}
c_{3} c_{2} & -s_{3} & 0 \\
s_{3} c_{2} & c_{3} & 0 \\
-s_{2} & 0 & 1
\end{array}\right)\left(\begin{array}{c}
\phi_{\lambda^{k}}^{1} \\
\phi_{\lambda^{k}}^{2} \\
\phi_{\lambda^{k}}^{3}
\end{array}\right) \equiv \mathbf{W}(\vec{\phi})\left(\begin{array}{c}
\phi_{\lambda^{k}}^{1} \\
\phi_{\lambda^{k}}^{2} \\
\phi_{\lambda^{k}}^{3}
\end{array}\right)
$$

This defines the molecule's intrinsic angular velocity by $\vec{\Omega} \equiv \dot{\vec{\theta}} \equiv \mathbf{W}(\vec{\phi}) \dot{\vec{\phi}}$. In our previous report, ${ }^{83}$ from the total of nine 
algebraic equations of (A13) a suitable set of three independent relations was selected and solved for $\vec{\theta}_{\lambda^{k}}$. However, we have recently investigated a more efficient way to accomplish this. Applying inner products with $\mathbf{e}_{21}, \mathbf{v}$, and $\mathbf{u}$ to both sides of eq. (A13), we obtain

$$
\vec{\theta}_{\lambda^{k}} \cdot \mathbf{e}_{21}=\mathbf{u} \cdot \frac{\partial \mathbf{v}}{\partial x_{\lambda}^{k}}, \vec{\theta}_{\lambda^{k}} \cdot \mathbf{v}=\mathbf{e}_{21} \cdot \frac{\partial \mathbf{u}}{\partial x_{\lambda}^{k}}, \vec{\theta}_{\lambda^{k}} \cdot \mathbf{u}=\mathbf{v} \cdot \frac{\partial \mathbf{e}_{21}}{\partial x_{\lambda}^{k}}
$$

This means $\vec{\theta}_{\lambda^{k}}$ is expressible by

$$
\vec{\theta}_{\lambda^{k}}=\left(\mathbf{u} \cdot \frac{\partial \mathbf{v}}{\partial x_{\lambda}^{k}}\right) \mathbf{e}_{21}+\left(\mathbf{e}_{21} \cdot \frac{\partial \mathbf{u}}{\partial x_{\lambda}^{k}}\right) \mathbf{v}+\left(\mathbf{v} \cdot \frac{\partial \mathbf{e}_{21}}{\partial x_{\lambda}^{k}}\right) \mathbf{u}
$$

as far as $\left\{\mathbf{e}_{21}, \mathbf{v}, \mathbf{u}\right\}$ is complete and orthonormal, which is true for nonlinear $\mathbf{x}_{i_{1}}, \mathbf{x}_{i_{2}}$, and $\mathbf{x}_{i_{3}}$. Differentiating this with respect to $x_{\nu}^{j}$ and using eq. (A13), we obtain

$$
\begin{array}{r}
\vec{\theta}_{\nu^{j} \lambda^{k}}=\left[\mathbf{u} \cdot \frac{\partial^{2} \mathbf{v}}{\partial x_{\nu}^{j} \partial x_{\lambda}^{k}}+\left(\mathbf{e}_{21} \cdot \frac{\partial \mathbf{v}}{\partial x_{\nu}^{j}}\right)\left(\mathbf{e}_{21} \cdot \frac{\partial \mathbf{u}}{\partial x_{\lambda}^{k}}\right)\right] \mathbf{e}_{21} \\
+\left[\mathbf{e}_{21} \cdot \frac{\partial^{2} \mathbf{u}}{\partial x_{\nu}^{j} \partial x_{\lambda}^{k}}+\left(\mathbf{v} \cdot \frac{\partial \mathbf{u}}{\partial x_{\nu}^{j}}\right)\left(\mathbf{v} \cdot \frac{\partial \mathbf{e}_{21}}{\partial x_{\lambda}^{k}}\right)\right] \mathbf{v} \\
+\left[\mathbf{v} \cdot \frac{\partial^{2} \mathbf{e}_{21}}{\partial x_{\nu}^{j} \partial x_{\lambda}^{k}}+\left(\mathbf{u} \cdot \frac{\partial \mathbf{e}_{21}}{\partial x_{\nu}^{j}}\right)\left(\mathbf{u} \cdot \frac{\partial \mathbf{v}}{\partial x_{\lambda}^{k}}\right)\right] \mathbf{u}
\end{array}
$$

with $\vec{\theta}_{\nu^{j} \lambda^{k}} \equiv \partial^{2} \vec{\theta} / \partial x_{\nu}^{j} \partial x_{\lambda}^{k}$. From eq. (A14), B-matrix elements for external rotations are now obtained by

$$
\begin{gathered}
\vec{\phi}_{\lambda^{k}}=\mathbf{W}^{-1} \vec{\theta}_{\lambda^{k}} \\
\vec{\phi}_{\nu^{j} \lambda^{k}}=\mathbf{W}^{-1}\left(\vec{\theta}_{\nu^{j} \lambda^{k}}-\left[\frac{\partial \mathbf{W}}{\partial x_{\nu}^{j}}\right] \vec{\phi}_{\lambda^{k}}\right) .
\end{gathered}
$$

In general, it is true that $\vec{\phi}_{\nu^{j} \lambda^{k}}=\vec{\phi}_{\lambda^{k} \nu^{j}}$, while we may have $\vec{\theta}_{\nu^{j} \lambda^{k}} \neq \vec{\theta}_{\lambda^{k} \nu^{j}}$.

For computational efficiency, instead of directly applying eqs. (A16) to (A18), we consider the values of $\vec{\theta}_{\lambda^{k}}$ in the MF frame, which from eqs. (A5) to (A7) are given by

$$
\vec{\theta}_{\lambda^{k} \mathrm{MF}} \equiv \Xi^{-1} \vec{\theta}_{\lambda^{k}}=\left(n_{1} \mathbf{u} \cdot \frac{\partial \mathbf{v}}{\partial x_{\lambda}^{k}} \quad n_{2} \mathbf{e}_{21} \cdot \frac{\partial \mathbf{u}}{\partial x_{\lambda}^{k}} \quad n_{3} \mathbf{v} \cdot \frac{\partial \mathbf{e}_{21}}{\partial x_{\lambda}^{k}}\right)_{\mathrm{MF}}^{T}
$$

Then, the expression for $\vec{\phi}_{\lambda^{k}}$ is simplified as

$$
\begin{aligned}
\vec{\phi}_{\lambda^{k}}= & \mathbf{W}^{-1} \mathbf{Z Z}^{-1} \vec{\theta}_{\lambda^{k}}=\mathbf{W}^{-1} \mathbf{Z} \vec{\theta}_{\lambda^{k} \mathrm{MF}} \\
& =\left(\begin{array}{lll}
\theta_{\lambda^{k} \mathrm{MF}}^{1}+s_{2} \tau_{\lambda^{k}} / c_{2} & c_{1} \theta_{\lambda^{k} \mathrm{MF}}^{2}-s_{1} \theta_{\lambda^{k} \mathrm{MF}}^{3} & \tau_{\lambda^{k}} / c_{2}
\end{array}\right)^{T}
\end{aligned}
$$

with

$$
\tau_{\lambda^{k}} \equiv s_{1} \theta_{\lambda^{k} \mathrm{MF}}^{2}+c_{1} \theta_{\lambda^{k} \mathrm{MF}}^{3}
$$

Next, we consider the fact that the involved derivatives with respect to the three atomic positions $\mathbf{x}_{i_{1}}, \mathbf{x}_{i_{2}}$, and $\mathbf{x}_{i_{3}}$, can be computed from derivatives with respect to the two bond vectors $\mathbf{a} \equiv$ $\mathbf{x}_{21}$ and $\mathbf{b} \equiv \mathbf{x}_{23}$ such as

$$
\partial \vec{\phi} / \partial x_{i_{1}}^{k}=\vec{\phi}_{a^{k}}, \partial \vec{\phi} / \partial x_{i_{2}}^{k}=-\vec{\phi}_{a^{k}}-\vec{\phi}_{b^{k}}, \quad \partial \vec{\phi} / \partial x_{i_{3}}^{k}=\vec{\phi}_{b^{k}}
$$

Thus, eq. (A21) for the first order B-matrix elements splits into

$$
\begin{gathered}
\vec{\phi}_{a^{k}}=\left(\begin{array}{lll}
\theta_{a^{k} \mathrm{MF}}^{1}+\frac{s_{2}}{c_{2}} \tau_{a^{k}} & c_{1} \theta_{a^{k} \mathrm{MF}}^{2}-s_{1} \theta_{a^{k} \mathrm{MF}}^{3} & \frac{1}{c_{2}} \tau_{a^{k}}
\end{array}\right)^{T} \\
\vec{\phi}_{b^{k}}=\left(\begin{array}{lll}
\theta_{b^{k} \mathrm{MF}}^{1} & 0 & 0
\end{array}\right)^{T} .
\end{gathered}
$$

Elements in the right-hand side of these can be efficiently computed by

$$
\begin{gathered}
\vec{\theta}_{a^{k} \mathrm{MF}}=\frac{1}{r_{21}}\left(\begin{array}{lll}
-n_{1} \frac{\cos \varphi_{13}}{\sin \varphi_{13}} u^{k} & -n_{2} u^{k} & n_{3} v^{k}
\end{array}\right)_{\mathrm{MF}}^{T} \\
\vec{\theta}_{b^{k} \mathrm{MF}}=\frac{1}{r_{23}}\left(\begin{array}{ccc}
\frac{n_{1}}{\sin \varphi_{13}} u^{k} & 0 & 0
\end{array}\right)_{\mathrm{MF}}^{T} \\
\tau_{\mathbf{a}}=\left(\begin{array}{llll}
\tau_{a^{1}} & \tau_{a^{2}} & \tau_{a^{3}}
\end{array}\right)_{\mathrm{MF}}^{T}=\frac{1}{r_{21}}\left(\begin{array}{lll}
-n_{1} s_{3} & n_{1} c_{3} & 0
\end{array}\right)_{\mathrm{MF}}^{T} \\
\tau_{\mathbf{b}}=\left(\begin{array}{lll}
\tau_{b^{1}} & \tau_{b^{2}} & \tau_{b^{3}}
\end{array}\right)_{\mathrm{MF}}^{T}=\mathbf{0}_{\mathrm{MF}},
\end{gathered}
$$

with

$$
\begin{aligned}
\mathbf{u}_{a^{k}} \equiv \frac{\partial \mathbf{u}}{\partial a^{k}} & =\frac{1}{r_{21} \sin \varphi_{13}}\left\{\hat{\mathbf{k}} \times \mathbf{e}_{23}+\left(\mathbf{u} \times \mathbf{e}_{23}\right)^{k} \mathbf{u}\right\} \\
\mathbf{u}_{b^{k}} & \equiv \frac{\partial \mathbf{u}}{\partial b^{k}}=\frac{1}{r_{23} \sin \varphi_{13}}\left\{\mathbf{e}_{21} \times \hat{\mathbf{k}}-\nu^{k} \mathbf{u}\right\} .
\end{aligned}
$$

Thus, the expression for $\phi_{a^{k}}^{2}$ in eq. (A24) is further simplified as

$$
\phi_{\mathbf{a}}^{2}=\left(\begin{array}{lll}
-s_{2} \tau_{a^{2}} & s_{2} \tau_{a^{1}} & -n_{1} c_{2} / r_{21}
\end{array}\right)^{T}
$$

Expressions for higher order derivatives of $\mathbf{u}$ with respect to the bond vectors can be found elsewhere, ${ }^{89}$ which is useful to compute nonzero elements of $\mathbf{B}_{2}$ for the external rotations.

Similarly, all nonzero expressions for the second order derivatives of $\vec{\phi}$ with respect to the two bond vectors are listed by

$$
\phi_{a^{j} a^{k}}^{1}=\theta_{a^{j} a^{k} \mathrm{MF}}^{1}+\frac{s_{2}}{c_{2}}\left(\tau_{a^{j} a^{k}}+\frac{1}{s_{2}} \phi_{a^{j}}^{2} \phi_{a^{k}}^{3}\right)
$$




$$
\begin{gathered}
\phi_{a^{j} a^{1}}^{2}=-c_{2} \phi_{a^{j}}^{2} \tau_{a^{2}}-s_{2} \tau_{a^{j} a^{2}} \\
\phi_{a^{j} a^{2}}^{2}=c_{2} \phi_{a^{\prime}}^{2} \tau_{a^{1}}+s_{2} \tau_{a^{j} a^{1}} \\
\phi_{a^{j} a^{3}}^{2}=-\frac{1}{r_{21}}\left(e_{21}^{3} \phi_{a^{j}}^{2}+e_{21}^{j} \phi_{a^{3}}^{2}\right) \\
\phi_{a^{j} a^{k}}^{3}=\frac{1}{c_{2}}\left(\tau_{a^{j} a^{k}}+s_{2} \phi_{a^{\prime}}^{2} \phi_{a^{k}}^{3}\right) \\
\phi_{b^{i} b^{k}}^{1}=\theta_{b^{j} b^{k} \mathrm{MF}}^{1} \\
\phi_{b^{j} a^{k}}^{1}=\phi_{a^{k} b^{j}}^{1}=\theta_{a^{k} b^{j} \mathrm{MF}}^{1}=\theta_{b^{j} a^{k} \mathrm{MF}}^{1} .
\end{gathered}
$$

Here, the necessary expressions for $\vec{\theta}_{a^{j} a^{k} \mathrm{MF}}, \vec{\theta}_{b^{j} b^{k} \mathrm{MF}}, \vec{\theta}_{b^{j} a^{k} \mathrm{MF}}$, and $\tau_{a^{j} a^{k}} \equiv \partial \tau_{a^{k}} \partial \partial a^{j}$ are found to be

$$
\begin{aligned}
& \tau_{a^{j} \mathbf{a}}=\left(\begin{array}{lll}
\tau_{a^{j} a^{1}} & \tau_{a^{j} a^{2}} & \tau_{a^{j} a^{3}}
\end{array}\right)_{\mathrm{MF}}^{T} \\
& =\left(\begin{array}{lll}
-\tau_{a^{2}} \phi_{a^{j}}^{3}-\frac{e_{21}^{j}}{r_{21}} \tau_{a^{1}} & \tau_{a^{1}} \phi_{a^{j}}^{3}-\frac{e_{21}^{j}}{r_{21}} \tau_{a^{2}} & 0
\end{array}\right)_{\mathrm{MF}}^{T} \\
& \theta_{a^{\prime} a^{k} \mathrm{MF}}^{1}=\frac{-n_{1}}{r_{21}^{2} \sin ^{2} \varphi_{13}}\left\{v^{j} u^{k}+v^{k} u^{j}-\sin \varphi_{13}\left[\cos \varphi_{13} e_{21}^{j} u^{k}+e_{23}^{k} u^{j}\right]\right\} \\
& =\frac{1}{r_{21}}\left\{n_{3} v^{j} \theta_{a^{k} \mathrm{MF}}^{2}-\left(e_{21}^{j}-\frac{\cos \varphi_{13}}{\sin \varphi_{13}} v^{j}\right) \theta_{a^{k} \mathrm{MF}}^{1}\right. \\
& \left.-\left\{e_{21}^{k}-\frac{\cos \varphi_{13}}{\sin \varphi_{13}} v^{k}\right) \theta_{a^{j} \mathrm{MF}}^{1}\right\} \\
& \theta_{a^{j} a^{k} \mathrm{MF}}^{2}=\frac{n_{2}}{r_{21}^{2} \sin \varphi_{13}}\left\{\sin \varphi_{13} e_{21}^{j} u^{k}-\left(\mathbf{u} \times \mathbf{e}_{23}\right)^{k} u^{j}\right\} \\
& =\frac{1}{r_{21}}\left\{n_{3} v^{k} \theta_{a^{j} \mathrm{MF}}^{1}-e_{21}^{j} \theta_{a^{k} \mathrm{MF}}^{2}-e_{21}^{k} \theta_{a^{j} \mathrm{MF}}^{2}\right\} \\
& \theta_{a^{j} a^{k} \mathrm{MF}}^{3}=\frac{n_{3}}{r_{21}^{2} \sin \varphi_{13}}\left\{e_{21}^{j} e_{23}^{k}-\cos \varphi_{13} \delta^{j k}\right. \\
& \left.+\left(\mathbf{u} \times \mathbf{e}_{23}\right)^{j} v^{k}-\sin \varphi_{13}\left[e_{21}^{j} v^{k}+e_{21}^{k} v^{j}\right]\right\} \\
& =\frac{1}{r_{21}}\left\{n_{2} u^{k} \theta_{a^{j} \mathrm{MF}}^{1}-e_{21}^{j} \theta_{a^{k} \mathrm{MF}}^{3}-e_{21}^{k} \theta_{a^{i} \mathrm{MF}}^{3}\right\}
\end{aligned}
$$

$$
\begin{gathered}
\theta_{b^{j} b^{k} \mathrm{MF}}^{1}=\frac{-n_{1}}{r_{23}^{2} \sin ^{2} \varphi_{13}}\left\{v^{j} u^{k}+v^{k} u^{j}\right\}\left[\text { since } \mathbf{v}_{b^{j}} \cdot \mathbf{u}_{b^{k}}=\mathbf{e}_{21} \cdot\left(\mathbf{u}_{b^{k}} \times \mathbf{u}_{b^{j}}\right)\right. \\
=0]=\frac{-1}{r_{23} \sin \varphi_{13}}\left\{v^{j} \theta_{b^{k} \mathrm{MF}}^{1}+v^{k} \theta_{b^{j} \mathrm{MF}}^{1}\right\}
\end{gathered}
$$

$$
\begin{aligned}
\theta_{b^{j} a^{k} \mathrm{MF}}^{1} & =\frac{n_{1}}{r_{21} r_{23} \sin ^{2} \varphi_{13}}\left\{\cos \varphi_{13}\left[v^{j} u^{k}+v^{k} u^{j}\right]-\sin \varphi_{13} e_{21}^{j} u^{k}\right\} \\
& =\frac{1}{r_{23} \sin \varphi_{13}}\left\{n_{3} e_{21}^{j} \theta_{a^{k} \mathrm{MF}}^{2}-v^{j} \theta_{a^{k} \mathrm{MF}}^{1}-v^{k} \theta_{a^{j} \mathrm{MF}}^{1}\right\} .
\end{aligned}
$$

We have used $\mathbf{u}_{b^{j}} \cdot\left(\mathbf{u}_{a^{k}} \times \mathbf{e}_{21}\right)=0$ and $\mathbf{v} \cdot(\mathbf{j} \times \mathbf{k})=u^{j} e^{k}{ }_{21}-$ $u^{k} e^{j}{ }_{21}$ in deriving the above equations.

These two direct methods of computing B-matrix elements for external rotations, viz., one from using eqs. (A10)-(A12) and the other given by eqs. (A24), (A25), and (A33)-(A39), are consistent with each other. We have verified the correctness of these analytical formulas up to second order by computational results of numerical differentiations of eqs. (A10)-(A12). The corresponding expressions for elements of $\mathbf{A}$ and $\mathbf{A}_{2}$ can be found elsewhere. ${ }^{82}$

\section{External Rotations with Four Parameters}

The above treatment of external rotations has a singularity problem for $\phi^{2}= \pm \pi / 2$ [see eqs. (A10)-(A12) or (A24)], and an artificial remedy is needed whenever this situation happens during simulation processes. As a way to avoid this problem, external rotations are also commonly represented by the Euler quaternion parameters $\left(e^{0} e^{1} e^{2} e^{3}\right)=\left(e^{0} \mathbf{e}\right)$, with a normalization condition of

$$
e^{0} e^{0}+\mathbf{e} \cdot \mathbf{e}=1
$$

In terms of these parameters, the rotation matrix in real three dimensions is given by ${ }^{97}$

$$
\begin{aligned}
& \Xi\left(e^{0}, \mathbf{e}\right) \\
& =\left(\begin{array}{ccc}
e^{0} e^{0}-\mathbf{e} \cdot \mathbf{e}+2 e^{1} e^{1} & 2 e^{1} e^{2}-2 e^{0} e^{3} & 2 e^{1} e^{3}+2 e^{0} e^{2} \\
2 e^{1} e^{2}+2 e^{0} e^{3} & e^{0} e^{0}-\mathbf{e} \cdot \mathbf{e}+2 e^{2} e^{2} & 2 e^{2} e^{3}-2 e^{0} e^{1} \\
2 e^{1} e^{3}-2 e^{0} e^{2} & 2 e^{2} e^{3}+2 e^{0} e^{1} & e^{0} e^{0}-\mathbf{e} \cdot \mathbf{e}+2 e^{3} e^{3}
\end{array}\right) .
\end{aligned}
$$

During our simulations for an isolated octane molecule in Application to an $n$-Octane Molecule, we have used the former method and encountered no singular cases probably due to short simulation time range. We suggest using the conventional representation with three parameters for large molecules and the Euler representation with quaternion parameters for small molecules.

Considering the diagonal sum of this matrix $\operatorname{tr}(\Xi)$ with eqs. (A4)-(A7), an expression to determine $e^{0}$ is found to be

$$
4 e^{0} e^{0}-1=e_{21}^{1} e_{21 \mathrm{MF}}^{1}+v^{2} v_{\mathrm{MF}}^{2}+v^{3} v_{\mathrm{MF}}^{3}=n_{1} e_{21}^{1}+n_{2} v^{2}+n_{3} u^{3}
$$


and the three parameters for $\mathbf{e}$ can be determined from

$$
\begin{aligned}
& 4 e^{0} e^{1}=\Xi_{32}-\Xi_{23}=n_{2} v^{3}-n_{3} u^{2} \\
& 4 e^{0} e^{2}=\Xi_{13}-\Xi_{31}=n_{3} u^{1}-n_{1} e_{21}^{3} \\
& 4 e^{0} e^{3}=\Xi_{21}-\Xi_{12}=n_{1} e_{21}^{2}-n_{2} v^{1} .
\end{aligned}
$$

The corresponding B-matrix elements (e.g., $e_{\lambda^{k}}^{0} \equiv \partial e^{0} / \partial x_{\lambda}^{k}$, $\mathbf{e}_{\lambda^{k}} \equiv \partial \mathbf{e} / \partial x_{\lambda}^{k}, e_{\nu^{j} \lambda^{k}}^{0} \equiv \partial^{2} e^{0} / \partial x_{\nu}^{j} \partial x_{\lambda}^{k}$, or $\left.\mathbf{e}_{\nu^{j} \lambda^{k}} \equiv \partial^{2} \mathbf{e} / \partial x_{\nu}^{j} \partial x_{\lambda}^{k}\right)$ can be computed by either analytical or numerical differentiations of eqs. (A48)-(A51) with respect to atomic coordinates.

However, we have also investigated an alternative method similar to that presented in the previous section. Considering eq. (A47) and differentiating eq. (A4) with respect to atomic coordinates, we can obtain the same expression as (A13). But, in this case, the intrinsic angular momentum $\dot{\vec{\theta}}$ is defined so that $^{82}$

$$
\left(\begin{array}{c}
0 \\
\vec{\theta}_{\lambda^{k}}
\end{array}\right)=\mathbf{W}\left(\begin{array}{c}
e_{\lambda^{k}}^{0} \\
\mathbf{e}_{\lambda^{k}}
\end{array}\right) \equiv 2\left(\begin{array}{cccc}
e^{0} & e^{1} & e^{2} & e^{3} \\
-e^{1} & e^{0} & -e^{3} & e^{2} \\
-e^{2} & e^{3} & e^{0} & -e^{1} \\
-e^{3} & -e^{2} & e^{1} & e^{0}
\end{array}\right)\left(\begin{array}{c}
e_{\lambda^{k}}^{0} \\
e_{\lambda^{k}}^{1} \\
e_{\lambda^{k}}^{2} \\
e_{\lambda^{k}}^{3}
\end{array}\right)
$$

The desired first order B-matrix elements can be efficiently computed by

$$
\begin{aligned}
\left(\begin{array}{c}
e_{\lambda^{k}}^{0} \\
\mathbf{e}_{\lambda^{k}}
\end{array}\right) & =\mathbf{W}^{-1}\left(\begin{array}{cc}
1 & 0 \\
0 & \Xi
\end{array}\right)\left(\begin{array}{cc}
1 & 0 \\
0 & \Xi^{-1}
\end{array}\right)\left(\begin{array}{c}
0 \\
\vec{\theta}_{\lambda^{k}}
\end{array}\right) \\
& =\frac{1}{2}\left(\begin{array}{cccc}
e^{0} & -e^{1} & -e^{2} & -e^{3} \\
e^{1} & e^{0} & -e^{3} & e^{2} \\
e^{2} & e^{3} & e^{0} & -e^{1} \\
e^{3} & -e^{2} & e^{1} & e^{0}
\end{array}\right)\left(\begin{array}{c}
0 \\
\theta_{\lambda^{k} \mathrm{MF}}^{1} \\
\theta_{\lambda^{k} \mathrm{MF}}^{2} \\
\theta_{\lambda^{k} \mathrm{MF}}^{3}
\end{array}\right) \\
& =\frac{1}{2}\left(\begin{array}{c}
-\mathbf{e} \vec{\theta}_{\lambda^{k} \mathrm{MF}} \\
e^{0} \vec{\theta}_{\lambda^{k} \mathrm{MF}}+\mathbf{e} \times \vec{\theta}_{\lambda^{k} \mathrm{MF}}
\end{array}\right)
\end{aligned}
$$

By differentiating this with respect to atomic coordinates $x_{\nu}^{j}$, expressions for the second order B-matrix elements are found to be

$4 e_{\nu^{j} \lambda^{k}}^{0}=-\left(\vec{\theta}_{\nu^{j} \mathrm{MF}} \cdot \vec{\theta}_{\lambda^{k} \mathrm{MF}}\right) e^{0}-\mathbf{e} \cdot\left(2 \vec{\theta}_{\nu^{j} \lambda^{k} \mathrm{MF}}+\vec{\theta}_{\nu^{j} \mathrm{MF}} \times \vec{\theta}_{\lambda^{k} \mathrm{MF}}\right)$

$$
\begin{aligned}
& 4 \mathbf{e}_{\nu^{j} \lambda^{k}}=-\left(\vec{\theta}_{\nu^{j} \mathrm{MF}} \cdot \vec{\theta}_{\lambda^{k} \mathrm{MF}}\right) \mathbf{e}+e^{0}\left(2 \vec{\theta}_{\nu^{j} \lambda^{k} \mathrm{MF}}+\vec{\theta}_{\nu^{j} \mathrm{MF}} \times \vec{\theta}_{\lambda^{k} \mathrm{MF}}\right) \\
&+\mathbf{e} \times\left(2 \vec{\theta}_{\nu^{j} \lambda^{k} \mathrm{MF}}+\vec{\theta}_{\nu^{j} \mathrm{MF}} \times \vec{\theta}_{\lambda^{k} \mathrm{MF}}\right) .
\end{aligned}
$$

With eqs. (A26), (A27), and (A41)-(A45), the whole computation processes can also be facilitated by the derivatives with respect to the two bond vectors $\mathbf{a}=\mathbf{x}_{21}$ and $\mathbf{b}=\mathbf{x}_{23}$. Finally, the corresponding expressions for elements of $\mathbf{A}$ and $\mathbf{A}_{2}$ can be found elsewhere. ${ }^{82}$

\section{References}

1. Wilson E. B., Jr.; Decius, J. C.; Cross, P. C. Molecular Vibrations; McGraw-Hill: New York, 1955.

2. Califano, S. Vibrational States; Wiley: New York, 1976.

3. Krimm, S.; Bandekar, J. Adv Protein Chem 1986, 38, 181.

4. Ramachandran, G. N.; Sasisekharan, V. Adv Protein Chem 1968, 23, 283.

5. Burkert, U.; Allinger, N. L. In ACS Monograph 177; Caserio, M. C., Ed.; American Chemical Society: Washington, DC, 1982.

6. Némethy, G.; Gibson, K. D.; Palmer, K. A.; Yoon, C. N.; Paterlini, G.; Zagari, A.; Rumsey, S.; Scheraga, H. A. J Phys Chem 1992, 96, 6472 .

7. Li, Z.; Scheraga, H. A. Proc Natl Acad Sci USA 1987, 84, 6611.

8. Chang, G.; Guida, W. C.; Still, W. C. J Am Chem Soc 1989, 111, 4379 .

9. Dodd, L. R.; Boone, T. D.; Theodorou, D. N. Mol Phys 1993, 78, 961.

10. Gabb, H. A.; Lavery, R.; Prévost, C. J Comput Chem 1995, $16,667$.

11. Deem, M. W.; Bader, J. S. Mol Phys 1996, 87, 1245.

12. Jorgensen, W. L.; Tirado-Rives, J. J Phys Chem 1996, 100, 14508.

13. Gabb, H. A.; Prévost, C.; Bertucat, G.; Robert, C. H.; Lavery, R. J Comput Chem 1997, 18, 2001.

14. Dinner, A. R. J Comput Chem 2000, 21, 1132.

15. Ulmschneider, J. P.; Jorgensen, W. L. J Chem Phys 2003, 118, 4261.

16. Pulay, P. Mol Phys 1969, 17, 197.

17. Schlegel, H. B. J Comput Chem 1982, 3, 214.

18. Schlegel, H. B. Theor Chim Acta (Berl) 1984, 66, 333.

19. Fogarasi, G.; Zhou, X.; Taylor, P. W.; Pulay, P. J Am Chem Soc 1992, 114, 8191.

20. Pulay, P.; Fogarasi, G. J Chem Phys 1992, 96, 2856.

21. Peng, C.; Ayala, P. Y.; Schlegel, H. B.; Frisch, M. J. J Comput Chem 1996, 17, 49.

22. Baker, J.; Kessi, A.; Delley, B. J Chem Phys 1996, 105, 192.

23. Baker, J. J Comput Chem 1997, 18, 1079.

24. Paizs, B.; Fogarasi, G.; Pulay, P. J Chem Phys 1998, 109, 6571.

25. Farkas, O.; Schlegel, H. B. J Chem Phys 1998, 109, 7100.

26. Baker, J.; Kinghorn, D.; Pulay, P. J Chem Phys 1999, 110, 4986.

27. Paizs, B.; Baker, J.; Suhai, S.; Pulay, P. J Chem Phys 2000, 113, 6566.

28. Kudin, K. N.; Scuseria, G. E.; Schlegel, H. B. J Chem Phys 2001, 114, 2919.

29. Farkas, O.; Schlegel, H. B. J Mol Struct (Theochem) 2003, 666/667, 31.

30. Németh, K.; Challacombe, M. J Chem Phys 2004, 121, 2877.

31. Tuzun, R. E.; Noid, D. W.; Sumpter, B. G. J Chem Phys 1996, 105 , 5494.

32. Clary, D. C. J Chem Phys 2001, 114, 9725.

33. Abagyan, R.; Totrov, M.; Kuznetsov, D. J Comput Chem 1994, 15, 488 .

34. Totrov, M.; Abagyan, R. Proteins 1997, Suppl. 1, 215.

35. Fernández-Recio, J.; Totrov, M; Abagyan, R. Protein Sci 2002, 11, 280.

36. Clore, G. M.; Schwieters, C. D. J Am Chem Soc 2003, 125, 2902.

37. Fernández-Recio, J.; Totrov, M.; Abagyan, R. Proteins 2003, 52, 113. 
38. Amadei, A.; Linssen, A. B. M.; Berendsen, H. J. C. Proteins 1993, $17,412$.

39. Ryckaert, J. P.; Ciccotti, G.; Berendsen, H. J. C. J Comput Phys 1977, 23, 327.

40. Andersen, H. C. J Comput Phys 1983, 52, 24.

41. Gō, N.; Scheraga, H. A. J Chem Phys 1969, 51, 4751.

42. Fixman, M.; Kovac, J. J Chem Phys 1974, 61, 4939.

43. Fixman, M.; Kovac, J. J Chem Phys 1974, 61, 4950.

44. Fixman, M. J Chem Phys 1978, 69, 1527.

45. Pear, M. R.; Weiner, J. H. J Chem Phys 1979, 71, 212.

46. Wittenburg, J. Symposium on Dynamics of Multibody Systems (Munich, Germany, August 28-September 3, 1977); Magnus, K., Ed.; Springer-Verlag: Heidelberg, 1978; pp. 357.

47. Jerkovsky, W. J Guid Cont 1978, 1, 173.

48. Katz, H.; Walter, R.; Somorjai, R. L. Comput Chem 1979, 3, 25.

49. Noguti, T.; Gō, N. J Phys Soc Japan 1983, 52, 3283.

50. Noguti, T.; Gō, N. J Phys Soc Japan 1983, 52, 3685.

51. Abe, H.; Braun, W.; Noguti, T.; Gō, N. Comput Chem 1984, 8, 239.

52. Gibson, K. D.; Scheraga, H. A. J Comput Chem 1990, 11, 468.

53. Gibson, K. D.; Scheraga, H. A. J Comput Chem 1990, 11, 487.

54. He, S.; Scheraga, H. A. J Chem Phys 1998, 108, 271.

55. Bystroff, C. Protein Eng 2001, 14, 825.

56. Mazur, A. K.; Abagyan, R. A. J Biomol Struct Dyn 1989, 6, 815.

57. Abagyan, R. A.; Mazur, A. K. J Biomol Struct Dyn 1989, 6, 833.

58. Mazur, A. K.; Dorofeyev, V. E.; Abagyan, R. A. J Comput Phys 1991, 92, 261.

59. Dorofeyev, V. E.; Mazur, A. K. J Comput Phys 1993, 107, 359.

60. Mazur, A. K. J Comput Chem 1997, 11, 1354.

61. Kneller, G. R.; Hinsen, K. Phys Rev E 1994, 50, 1559.

62. Hinsen, K.; Kneller, G. R. Phys Rev E 1995, 52, 6868.

63. Bae, D.-S.; Haug, E. J Mech Struct Mach 1987, 15, 359.

64. Bae, D.-S.; Haug, E. J Mech Struct Mach 1987, 15, 481.

65. Rodriguez, G.; Jain, A.; Kreutz-Delgado, K. Int J Rob Res 1991, 10, 371.

66. Jain, A.; Vaidehi, N.; Rodriguez, G. J Comput Phys 1993, 106, 258.

67. Rice, L. M.; Brünger, A. T. Proteins 1994, 19, 277.

68. Mathiowetz, A. M.; Jain, A.; Karasawa, N.; Goddard W. A., III. Proteins 1994, 20, 227.

69. Turner, J.; Weiner, P.; Robson, B.; Venugopal, R.; Schubele H., III; Singh, R. J Comput Chem 1995, 16, 1271.
70. Vaidehi, N.; Jain, A.; Goddard W. A., III. J Phys Chem 1996, 100, 10508.

71. Stein, E. G.; Rice, L. M.; Brünger, A. T. J Magn Reson 1997, 124, 154.

72. Güntert, P.; Mumenthaler, C.; Wüthrich, K. J Mol Biol 1997, 273, 283.

73. Schwieters, C. D.; Clore, G. M. J Magn Reson 2001, 152, 288.

74. Rapaport, D. C. Phys Rev E 2002, 66, 011906.

75. Bertsch, R. A.; Vaidehi, N.; Chan, S. I.; Goddard W. A., III. Proteins $1998,33,343$.

76. Klepeis, J. L.; Floudas, C. A. Comput Chem Eng 2000, 24, 1761.

77. Zhang, C.; Hou, J.; Kim, S.-H. Proc Natl Acad Sci USA 2002, 99, 3581 .

78. Vaidehi, N.; Floriano, W. B.; Trabanino, R.; Hall, S. E.; Freddolino, P.; Choi, E. J.; Zamanakos, G.; Goddard W. A., III. Proc Natl Acad Sci USA 2002, 99, 12622.

79. Herrmann, T.; Güntert, P.; Wüthrich, K. J Mol Biol 2002, 319, 209.

80. Chen, J.; Im, W.; Brooks C. L., III. J Comput Chem 2005, 26, 1565.

81. Bardiaux, B.; Malliavin, T. E.; Nilges, M.; Mazur, A. K. J Biomol NMR 2006, 34, 153.

82. Lee, S.-H.; Palmo, K.; Krimm, S. Chem Phys 2001, 265, 63.

83. Lee, S.-H.; Palmo, K.; Krimm, S. Chem Phys Lett 2001, 342, 643.

84. Lee, S.-H.; Palmo, K.; Krimm, S. J Comput Phys 2005, 210, 171.

85. Pulay, P.; Paizs, B. Chem Phys Lett 2002, 353, 400.

86. Mazur, A. K. J Phys Chem B 1998, 102, 473.

87. Stocker, U.; Juchli, D.; van Gunsteren, W. F. Mol Simulat 2003, 29, 123.

88. Swope, W. C.; Andersen, H. C.; Berens, P. H.; Wilson, K. R. J Chem Phys 1982, 76, 637.

89. Lee, S. -H.; Palmo, K.; Krimm, S. J Comput Chem 1999, 20, 1067.

90. Axelsson, O. Iterative Solution Methods; Cambridge University Press: Cambridge, 1994.

91. Palmo, K.; Mirkin, N. G.; Krimm, S. J Phys Chem A 1998, 102, 6448.

92. Zhang, M. -Q.; Skeel, R. D. J Comput Chem 1995, 16, 365.

93. Allen, W. D.; Császár, A. G. J Chem Phys 1993, 98, 2983.

94. Eckart, C. Phys Rev 1935, 47, 552.

95. Wilson E. B., Jr.; Howard, J. B. J Chem Phys 1936, 4, 260.

96. Sayvetz, A. J Chem Phys 1939, 7, 383.

97. Evans, D. J. Mol Phys 1977, 34, 317. 